Groups Geom. Dyn. 6 (2012), 53-82

DOI $10.4171 / \mathrm{GGD} / 150$
Groups, Geometry, and Dynamics

(C) European Mathematical Society

\title{
Free products, orbit equivalence and measure equivalence rigidity
}

\author{
Aurélien Alvarez and Damien Gaboriau
}

\begin{abstract}
We study the analogue, in orbit equivalence, of free product decompositions and free indecomposability for countable groups. We introduce the (orbit equivalence invariant) notion of freely indecomposable $(\mathcal{F} \mathcal{I})$ standard probability measure preserving equivalence relations and establish a criterion to check it, namely non-hyperfiniteness and vanishing of the first $L^{2}$ Betti number. We obtain Bass-Serre rigidity results, i.e. forms of uniqueness in free product decompositions of equivalence relations with $(\mathcal{F} \mathcal{I})$ components. The main features of our work are weak algebraic assumptions and no ergodicity hypothesis for the components. We deduce, for instance, that a measure equivalence between two free products of non-amenable groups with vanishing first $\ell^{2}$-Betti numbers is induced by measure equivalences of the components. We also deduce new classification results in orbit equivalence and $\mathrm{II}_{1}$ factors.
\end{abstract}

Mathematics Subject Classification (2010). 37A20, 20 E06.

Keywords. Orbit equivalence, measure equivalence, free product decomposition, $L^{2}$-Betti numbers.

\section{Introduction}

Bass-Serre theory [Ser77] studies groups acting on trees and offers extremely powerful tools to understand their structure, together with a geometric point of view that illuminates several classical results on free product decompositions. For instance Kurosh's subgroup theorem [Kur34], that describes the subgroups in a free product of groups and, as a by-product, the essential uniqueness in free product decompositions into freely indecomposable subgroups, is much easier to handle via Bass-Serre theory.

In orbit equivalence theory, the notion of free products or freely independent standard equivalence relations introduced in [Gab00] proved to be useful in studying the cost of equivalence relations and for some classification problems. The purpose of our article is connected with the uniqueness condition in free product decompositions, in the measurable context. To this end, we will take full advantage of the recent work of the first named author [Alv08a], [Alv08b], who develops a Bass-Serre theory in 
this context. In particular, Theorem 3.1 and Theorem 3.2 will be crucial for our purpose.

Very roughly, the kind of results we are after claim that if a standard measured equivalence relation is decomposed in two ways into a free product of factors that are not further decomposable in an appropriate sense, then the factors are pairwise related. However, due to a great flexibility in decomposability, it appears that certain types of free decomposition, namely slidings (Definition 2.7) and slicings (Definition 2.6), are banal and somehow inessential (see Section 2.4). We thus start by clearing up the notion of a freely indecomposable ( $\mathcal{F} \mathcal{I}$ ) standard measured countable equivalence relation (Definition 4.5), ruling out inessential decompositions (Definition 4.1).

A countable group $\Gamma$ is said to be measurably freely indecomposable $(\mathcal{M} \mathcal{F} \mathcal{I})$ if all its free probability measure preserving (p.m.p.) actions produce freely indecomposable $(\mathcal{F} \mathcal{I})$ equivalence relations. As expected, a free product of two infinite groups is not $\mathcal{M F} \mathcal{I}$, and in fact none of its free p.m.p. actions is $\mathcal{F} \mathcal{I}$. The same holds for infinite amenable groups (cf. Corollary 4.7). On the other hand, freely indecomposable groups in the classical sense are not necessarily $\mathcal{M F} \mathcal{F}$, for instance the fundamental group of a closed orientable surface of genus $\geq 2$ (see Proposition 4.13). We now give a prototypical instance of our results:

Theorem 1.1. Consider two families of infinite countable $\mathcal{M F} \mathcal{F}$ groups $\left(\Gamma_{i}\right)_{i \in I}$ and $\left(\Lambda_{j}\right)_{j \in J}, I=\{1,2, \ldots, n\}, J=\{1,2, \ldots, m\}, n, m \in \mathbb{N}^{*} \cup\{\infty\}$. Consider two free probability measure preserving actions $\alpha$ and $\beta$ of the free products on standard Borel spaces whose restrictions to the factors $\alpha \mid \Gamma_{i}$ and $\beta \mid \Lambda_{j}$ are ergodic. If the actions $\alpha$ and $\beta$ are stably orbit equivalent,

$$
\left.\left.\underset{i \in I}{*} \Gamma_{i}\right) \curvearrowright^{\alpha}(X, \mu) \stackrel{\text { SOE }}{\sim} \underset{j \in J}{*} \Lambda_{j}\right) \curvearrowright^{\beta}(Y, v),
$$

then $n=m$ and there is a bijection $\theta: I \rightarrow J$ for which the restrictions are stably orbit equivalent,

$$
\alpha\left|\Gamma_{i} \stackrel{\text { SOE }}{\sim} \beta\right| \Lambda_{\theta(i)} .
$$

Of course, such a statement urges us to exhibit $\mathcal{M F} \mathcal{F}$ groups, and it appears that their class is quite large:

Theorem 1.2 (Corollary 4.20). Every non-amenable countable group $\Gamma$ with vanishing first $\ell^{2}$-Betti number $\left(\beta_{1}(\Gamma)=0\right)$ is measurably freely indecomposable (MFF $)$.

Recall that the $\ell^{2}$-Betti numbers are a sequence of numbers $\beta_{r}(\Gamma)$ defined by Cheeger-Gromov [CG86]: they are attached to each countable discrete group $\Gamma$ and that they have a general tendency to concentrate in a single dimension $r$ and to vanish in the other ones (see [BV97], [Lüc02]). The first $\ell^{2}$-Betti number vanishes for many "usual" groups, for instance amenable groups, direct products of infinite groups, lattices in $\mathrm{SO}(p, q)(p \cdot q \neq 2)$, lattices in $\mathrm{SU}(p, q)$, groups with Kazhdan's 
property $(\mathrm{T})$. It is worth noting that infinite Kazhdan's property $(\mathrm{T})$ groups also follow $\mathcal{M F I}$ from Adams-Spatzier [AS90], Th. 1.1 (see [Gab00], Ex. IV.12). The list of groups with vanishing $\beta_{1}$ may be continued, for instance, with the groups with an infinite finitely generated normal subgroup of infinite index, groups with an infinite normal subgroup with the relative property (T), amalgamated free products of groups with $\beta_{1}=0$ over an infinite subgroup, mapping class groups... On the other hand, for a free product of two (non trivial) groups we have $\beta_{1}\left(\Gamma_{1} * \Gamma_{2}\right)>0$ unless $\Gamma_{1}=\Gamma_{2}=\mathbb{Z} / 2 \mathbb{Z}$, in which case $\Gamma_{1} * \Gamma_{2}$ is amenable.

Results in the spirit of Theorem 1.1 were obtained as by-products of operator algebraic considerations in [IPP08], Cor. 0.5 , Cor. 7.8 , Cor. $7.8^{\prime}$, and also recently in [CH10], Cor. 6.7. Our results cover a large part of these corollaries. We will come back more precisely on the differences between these papers and ours, but an important issue is that they both require the ergodicity of the actions restricted to the factors and some particular algebraic assumptions on the groups.

We will extend our framework by introducing marginal free groups or relatives (recall that in Kurosh's theorem there are "vertex subgroups" and a free group) and, more seriously, by removing the ergodicity assumption on the actions of the factors; and both of these extensions prove to be necessary to handle with measure equivalence of groups (see [Gab05] for a survey on this notion introduced by M. Gromov). Recall that two countable groups $\Gamma$ and $\Lambda$ are measure equivalent (ME), in symbols:

$$
\Gamma \underset{\kappa}{\mathrm{ME}} \Lambda
$$

if and only if they admit stably orbit equivalent (SOE) free p.m.p. actions. The real number $\kappa \in \mathbb{R}_{+}^{*}$ is called the generalized index or the compression constant according to whether one focuses on the classification of groups up to ME or on more operator algebraic aspects of orbit equivalence. Commensurable groups are ME, and the generalized index then coincides with the usual index for subgroups. It is proved in [Gab05], $\mathbf{P}_{\mathrm{ME}} \mathbf{6}$, p. 1814-1816) that measure equivalent groups with generalized index 1 induce measure equivalence of their free products:

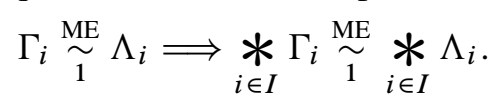

Our techniques allow us to settle a converse when the factors are $\mathcal{M} \mathcal{F} \mathcal{I}$. We observe that being $\mathcal{M F I}$ is a measure equivalence invariant (Proposition 4.13), and before stating our ME result, we consider the following striking example. It prevents us from being overoptimistic or expecting a bijective correspondence between the factors.

Example 1.3. If $\Gamma_{1}^{\prime} \triangleleft \Gamma_{1}$ and $\Gamma_{2}^{\prime} \triangleleft \Gamma_{2}$ are two normal subgroups of finite index $\kappa$ such that $\Gamma_{1} / \Gamma_{1}^{\prime} \simeq \Gamma_{2} / \Gamma_{2}^{\prime} \simeq K$, then the following groups are ME with $\Gamma_{1} * \Gamma_{2}$ with generalized index $\kappa$ and they satisfy:

$$
\Gamma_{1}^{\prime} * \underbrace{\Gamma_{2} * \Gamma_{2} * \cdots * \Gamma_{2}}_{\kappa \text { copies }} \underset{1}{\stackrel{\mathrm{ME}}{\sim}} \underbrace{\Gamma_{1} * \Gamma_{1} * \cdots * \Gamma_{1}}_{\kappa \text { copies }} * \Gamma_{2}^{\prime} \underset{1}{\stackrel{\mathrm{ME}}{\sim}} \Gamma_{1}^{\prime} * \Gamma_{2}^{\prime} * \mathbf{F}_{\kappa-1}
$$


where $\mathbf{F}_{p}$ is the free group on $p$ generators. In fact these three groups are even mutually commensurable with finite kernels and generalized index 1, since they appear as the kernels of the three natural epimorphisms $\Gamma_{1} * \Gamma_{2} \rightarrow K$.

Theorem 1.1 ensures that such "pathologies" are ruled out by adding ergodicity assumptions on the actions of the factors. Explicit actions witnessing these measure equivalences are easily produced by suspension, and the fact that they are not ergodic when restricted to some factors is not at all incidental (and the above Example 1.3 may be better understood). We are able, from Theorem 5.1 (see Remark 5.3), to localize some constraints on the failure of ergodicity, for instance for any action witnessing a measure equivalence between the following commensurable groups:

Corollary 1.4. Assume that $\Gamma_{1}, \Gamma_{2}$ are $\mathcal{M F I}$ and not $M E$, and that $\Gamma_{1}^{\prime}$ has finite index $\geq 2$ in $\Gamma_{1}$. Then, for any stably orbit equivalent free actions $\Gamma_{1} * \Gamma_{2} \curvearrowright^{\alpha} X$ and $\Gamma_{1}^{\prime} * \Gamma_{2} * \Gamma_{2} * \cdots * \Gamma_{2} \curvearrowright^{\beta} Y$, the restriction $\alpha \mid \Gamma_{2}$ is not ergodic.

We are now in position to state our general measure equivalence result:

Theorem 1.5 (ME Bass-Serre rigidity). Consider two families of infinite countable $\mathcal{M} \mathcal{F} \mathcal{I}$ groups (for instance non-amenable with vanishing first $\ell^{2}$-Betti number) $\left(\Gamma_{i}\right)_{i \in I}$ and $\left(\Lambda_{j}\right)_{j \in J}, I=\{1,2, \ldots, n\}, J=\{1,2, \ldots, m\}, n, m \in \mathbb{N}^{*} \cup\{\infty\}$. If their free products are measure equivalent,

$$
\underset{i \in I}{*} \Gamma_{i} \stackrel{\mathrm{ME}}{\sim} \underset{j \in J}{*} \Lambda_{j}
$$

then there are two maps $\theta: I \rightarrow J$ and $\theta^{\prime}: J \rightarrow I$ such that

$$
\Gamma_{i} \stackrel{\mathrm{ME}}{\sim} \Lambda_{\theta(i)} \text { and } \Lambda_{j} \stackrel{\mathrm{ME}}{\sim} \Gamma_{\theta^{\prime}(j)} .
$$

Moreover, if $\Gamma_{0}, \Lambda_{0}$ are two groups in the ME classes of some free groups, then the same conclusion holds under the assumption

$$
\underset{i \in I}{*} \Gamma_{i} * \Gamma_{0} \stackrel{\mathrm{ME}}{\sim} \underset{j \in J}{*} \Lambda_{j} * \Lambda_{0}
$$

Observe that we do not assume the generalized index $\kappa=1$. Would we do so, we would not get $\kappa=1$ in the conclusion as Example 1.3 again indicates. Also observe that the groups $\Gamma_{0}, \Lambda_{0}$ do not appear in the conclusion.

Let us mention a recent Bass-Serre rigidity result obtained by Sako via $C^{*}$-algebra techniques, and dealing with free products with amalgamation of direct products of non-amenable exact groups over a common amenable subgroup [Sak10].

It is interesting to observe that one may combine our theorem 1.5 for free products with Monod-Shalom's Theorem 1.16 [MS06] for direct products. Using the facts that a free product of infinite groups belongs to their class $C_{\text {reg }}$, and that a direct product of non-amenable groups is $\mathcal{M F} \mathcal{F}$, we get the following type of results: 
Corollary 1.6. Assume $\Gamma$ is either $\Gamma_{i}$, or

i) a finite direct product of non-trivial free products of torsion-free $\mathcal{M F I}$ groups

ii) a free product of non-trivial finite direct products of torsion free groups $\Gamma_{i}$ in the class $C_{\mathrm{reg}}$.

If $\Gamma$ is measure equivalent with a group $\Lambda$ of the same kind, then the elementary pieces $\Gamma_{i}$ of $\Gamma$ define the same set of ME-classes as those of $\Lambda$.

Of course, this construction can be iterated by taking alternatively free or finite direct products of groups $\Gamma_{i}$ as in i) or ii) above, according to whether the first operation is a free or a direct product. A measure equivalence with a group $\Lambda$ of the same kind entails measure equivalences between the elementary pieces. Notice that the number of iterations follows the same for $\Gamma$ and $\Lambda$.

The above Theorem 1.5 is essentially a consequence of the following SOE Theorem 1.8 (see Theorem 5.1 for the more general measured equivalence relations statement). We continue with similar data. In the SOE context, the role of free groups is played by treeability (see [Ada88], [Gab00] or Section 2.3 for more on this notion). Recall that it follows from [Hjo06] that a group $\Gamma$ is $\mathrm{ME}$ with a free group if and only if it admits a free p.m.p. treeable action (see [Gab05], $\mathbf{P}_{\mathrm{ME}} \mathbf{8}$ ). A group is said strongly treeable if all its free p.m.p. actions are treeable. This is for instance the case of the amenable groups (even finite or even trivial) or the free products of amenable groups (e.g. free groups). We have no example of a group that is ME with a free group but that is not strongly treeable.

We display separately the following framework that will be in use in the next results:

Framework 1.7. Let

- $\left(\Gamma_{p}\right)_{p \in P}$ and $\left(\Gamma_{p^{\prime}}^{\prime}\right)_{p^{\prime} \in P^{\prime}}, P=\{1,2, \ldots, n\}, P^{\prime}=\left\{1,2, \ldots, n^{\prime}\right\}, n, n^{\prime} \in$ $\mathbb{N}^{*} \cup\{\infty\}$ be two families of infinite countable groups,

$-\Gamma_{0}, \Gamma_{0}^{\prime}$ be two countable groups.

Assume $\alpha$ and $\alpha^{\prime}$ are two free p.m.p. stably orbit equivalent actions

$$
\left.\left.\underset{p \in P}{*} \Gamma_{p} * \Gamma_{0}\right) \curvearrowright^{\alpha}(X, \mu) \stackrel{\operatorname{SOE}}{\sim} \underset{p^{\prime} \in P^{\prime}}{*} \Gamma_{p^{\prime}}^{\prime} * \Gamma_{0}^{\prime}\right) \curvearrowright^{\alpha^{\prime}}\left(X^{\prime}, \mu^{\prime}\right)
$$

of the free products on standard Borel spaces, such that

- the restrictions $\alpha \mid \Gamma_{p}$ and $\alpha^{\prime} \mid \Gamma_{p^{\prime}}^{\prime}$ are freely indecomposable (F्FI) (for $p, p^{\prime} \in$ $\left.P, P^{\prime}\right)$,

- the restrictions $\alpha \mid \Gamma_{0}$ and $\alpha^{\prime} \mid \Gamma_{0}^{\prime}$ are treeable.

Theorem 1.8 (SOE Bass-Serre rigidity). If $\alpha$ and $\alpha^{\prime}$ are two SOE actions as in Framework 1.7, then up to countable partitions, the components are in one-to-one correspondence in the following sense. There exist 
1. for each $p \in P$, a measurable $\alpha \mid \Gamma_{p}$-invariant partition $X=\coprod_{k \in K(p)} X_{k}$,

2. for each $p^{\prime} \in P^{\prime}$, a measurable $\alpha^{\prime} \mid \Gamma_{p^{\prime}}^{\prime}$-invariant partition $X^{\prime}=\coprod_{k^{\prime} \in K^{\prime}\left(p^{\prime}\right)} X_{k^{\prime}}^{\prime}$,

3. a bijection $\theta: \coprod_{p \in P} K(p) \rightarrow \coprod_{p^{\prime} \in P^{\prime}} K^{\prime}\left(p^{\prime}\right)$ between the index sets

according to which the restrictions of the actions to the factors and the subsets are SOE:

$$
\forall k \in \coprod_{p \in P} K(p), \quad \alpha\left|\left(\Gamma_{k} \times X_{k}\right) \stackrel{\text { SOE }}{\sim} \alpha^{\prime}\right|\left(\Gamma_{\theta(k)}^{\prime} \times X_{\theta(k)}^{\prime}\right)
$$

with the obvious notational conventions: $\Gamma_{k}:=\Gamma_{p}$ for the unique $p \in P$ such that $k \in K(p)$, and $\Gamma_{\theta(k)}^{\prime}:=\Gamma_{p^{\prime}}^{\prime}$ for the unique $p^{\prime} \in P^{\prime}$ such that $\theta(k) \in K^{\prime}\left(p^{\prime}\right)$.

Observe that under ergodic assumptions on the actions restricted to the factors, the invariant partitions turn out to be trivial and $\theta$ gives a bijective correspondence between the original index sets. Ergodicity on one side may force the same situation:

Corollary 1.9. Consider two SOE actions $\alpha$ and $\alpha^{\prime}$ as in Framework 1.7. Assume that $n \leq n^{\prime}<\infty$ and that the restrictions of the actions to the $\Gamma_{p}$-factors $\alpha \mid \Gamma_{p}$ are ergodic, $\forall p \in P$. Then the restrictions $\alpha^{\prime} \mid \Gamma_{p^{\prime}}^{\prime}$ are also ergodic, $n=n^{\prime}$ and $\theta$ gives a bijection $\theta: P \rightarrow P^{\prime}$. If moreover $\beta_{1}\left(\Gamma_{p}\right)=\beta_{1}\left(\Gamma_{p^{\prime}}^{\prime}\right)=0$ for all $p, p^{\prime} \in P, P^{\prime}$ and $\Gamma_{0}=\Gamma_{0}^{\prime}=\{1\}$, then the factors follow orbit equivalent $\alpha\left|\Gamma_{p} \stackrel{\mathrm{OE}}{\sim} \alpha^{\prime}\right| \Gamma_{\theta\left(p^{\prime}\right)}^{\prime}$.

We also get some consequences for ergodic components from Theorem 5.1. It is a banal observation that the number of ergodic components (let us denote it \#erg comp $(\sigma))$ of a single action $G \curvearrowright^{\sigma}(X, \mu)$ is invariant under stable orbit equivalence. We obtain a survival of this invariant for a restriction of an action to factors of a free product:

Corollary 1.10. Consider two SOE actions $\alpha$ and $\alpha^{\prime}$ as in Framework 1.7. Let $P_{1} \subset P$ and $P_{1}^{\prime} \subset P^{\prime}$ be the indices of those groups $\Gamma_{p}, \Gamma_{p^{\prime}}^{\prime}$ that are measure equivalent with $\Gamma_{1}$. Then the number of ergodic components of the restrictions are equal:

$$
\sum_{p \in P_{1}} \# \operatorname{erg} \operatorname{comp}\left(\alpha \mid \Gamma_{p}\right)=\sum_{p^{\prime} \in P_{1}^{\prime}} \# \operatorname{erg} \operatorname{comp}\left(\alpha^{\prime} \mid \Gamma_{p^{\prime}}^{\prime}\right)
$$

Even, the measures of the ergodic components become a SOE invariant, under a control of the self generalized indices. The set $I_{\mathrm{ME}}(\Gamma)$ of possible generalized indices $\kappa$ in measure equivalences between a group $\Gamma$ and itself $\Gamma \underset{\kappa}{\stackrel{\mathrm{ME}}{\sim}} \Gamma$ is an invariant of the ME class of $\Gamma$ (see [Gab02b] or [Gab05], $\left.\mathbf{P}_{\mathrm{ME}} \mathbf{1 7}\right)$. The condition $I_{\mathrm{ME}}(\Gamma)=\{1\}$ is obtained for instance when $\Gamma$ has an $\ell^{2}$-Betti number $\beta_{q}(\Gamma) \neq 0, \infty$ [Gab02a]. For sake of simplicity, we give a sample of the kind of statements that may be derived from Theorem 5.1 (see Theorem 6.4): 
Corollary 1.11. Assume that the $\left(\Gamma_{p}\right)_{p \in P}$ have vanishing $\beta_{1}$ and that $\Gamma_{0}$ is a free group. Assume that $\Gamma_{1}$ admits at least one $\ell^{2}$-Betti number $\beta_{q}\left(\Gamma_{1}\right)$ different from 0 and $\infty$ and that it is not measure equivalent with any of the other $\Gamma_{p}, p \neq 1$. If $\Theta$ is a SOE between two p.m.p. actions $\alpha$ and $\alpha^{\prime}$ of $\left(*_{p \in P} \Gamma_{p} * \Gamma_{0}\right)$, then $\Theta$ is in fact an $O E$ and the restrictions to $\Gamma_{1}$ are $O E$. In particular, they have the same measure space of ergodic components.

Corollary 1.12. Let $\Gamma_{0}=\mathbf{F}_{2}$ and $\Gamma_{1}=\mathbf{F}_{3} \times \mathbf{F}_{3}$. Consider a one-parameter family offree p.m.p. actions $\Gamma_{0} * \Gamma_{1} \curvearrowright^{\alpha_{s}}(X, \mu)$, where the restriction $\alpha_{s} \mid \Gamma_{1}$ has two ergodic components of respective measures $s, 1-s$. The actions $\alpha_{s}$ are not mutually stably orbit equivalent for $s \in[0,1 / 2]$.

Recall that free p.m.p. group actions $\Gamma \curvearrowright^{\sigma}(X, \mu)$ define finite von Neumann algebras by the so called group-measure-space construction of Murray-von Neumann or von Neumann crossed product $L^{\infty}(X, \mu) \rtimes_{\sigma} \Gamma$. Stably orbit equivalent actions define stably isomorphic crossed-products, but the converse does not hold in general, and this leads to the following definition. Two free p.m.p. actions $\Gamma \curvearrowright^{\sigma}(X, \mu)$ and $\Gamma^{\prime} \curvearrowright^{\sigma^{\prime}}\left(X^{\prime}, \mu^{\prime}\right)$ are called von Neumann stably equivalent if there is $\kappa \in(0, \infty)$ such that $L^{\infty}(X, \mu) \rtimes_{\sigma} \Gamma \simeq\left(L^{\infty}\left(X^{\prime}, \mu^{\prime}\right) \rtimes_{\sigma^{\prime}} \Gamma^{\prime}\right)^{\kappa}$.

Both papers [IPP08], [CH10] establish rigidity phenomena in operator algebras and derive orbit equivalence results for the components of free products from an assumption of von Neumann stable equivalence on the actions. To this end, some strong algebraic constraints on the involved groups are imposed. More precisely in [IPP08], Cor. 0.5, Cor. 7.8, Cor. 7.8', the analysis relies on the notion of relative property (T) in von Neumann algebras introduced by S. Popa in [Pop06], and thus the groups $\Gamma_{p}, \Gamma_{p}^{\prime}$ (in the notation of Framework 1.7) are required to admit a non virtually abelian subgroup with the relative property (T) and some ICC-like and normal-like properties (for instance, they may be ICC property (T) groups) (see [IPP08], Assumption 7.6). In [CH10], Cor. 6.7, the operator algebraic notion involved is primality, so that the assumption on the groups $\Gamma_{p}, \Gamma_{p}^{\prime}$ is to be ICC non-amenable direct products of infinite groups. In both cases, they all satisfy $\beta_{1}=0$. As already mentioned, the actions restricted to the factors $\alpha \mid \Gamma_{p}$ and $\alpha^{\prime} \mid \Gamma_{p^{\prime}}^{\prime}$ are assumed to be ergodic. On the other hand, the assumption that the actions are SOE $\alpha \stackrel{\text { SOE }}{\sim} \alpha^{\prime}$ is replaced by the weaker one that $\alpha$ and $\alpha^{\prime}$ are von Neumann stably equivalent. All these results exploit the antagonism between free products and either various forms of property $(\mathrm{T})$, or direct product, or more generally in our case the vanishing of the first $\ell^{2}$-Betti number. Also, in [IPP08] the "marginal" groups $\Gamma_{0}, \Gamma_{0}^{\prime}$ are solely assumed to be a-T-menable (i.e. to have Haagerup property) a property that in turn is antagonist to property (T), while our $\Gamma_{0}, \Gamma_{0}^{\prime}$ are ME with a free group (antagonist to $\mathcal{F} \mathcal{I})$ and thus a-T-menable. Observe that there are $\mathcal{M} \mathcal{F} \mathcal{I}$ a-T-menable groups, thus able to play the role of a $\Gamma_{p}$ or a $\Gamma_{0}$ according to the approach.

On the other hand, it follows from [IPP08], Th. 7.17, Cor. 7.18, that von Neumann stable equivalence entails stable orbit equivalence, among the free p.m.p. actions of 
free products of (at least two) infinite groups, as soon as one of the two actions has the relative property (T) in the sense of [Pop06], Def. 4.1. Meanwhile, Theorem 1.2 of [Gab08] establishes that any free product of at least two infinite groups admits a continuum of relative property ( $\mathrm{T}$ ) von Neumann stably inequivalent ergodic free p.m.p. actions, whose restriction to each free product component is conjugate with any prescribed (possibly non-ergodic) action.

When injected in our context, this gives further classifications results for $\mathrm{II}_{1}$ factors. For instance:

Theorem 1.13. Let $\Gamma_{1}, \Gamma_{2}$ be non-ME, non-amenable groups with $\beta_{1}=0$. Assume $\beta_{q}\left(\Gamma_{1}\right) \neq 0, \infty$ for some $q>1$. The crossed-product II $I_{1}$ factors $M_{1} *_{A} M_{2}$ associated with the various ergodic relative property (T) free p.m.p. actions $\Gamma_{1} * \Gamma_{2} \curvearrowright^{\sigma}(X, \mu)$ are classified by the pairs $A \subset M_{1}$, and in particular by the isomorphism class of the centers $Z\left(M_{1}\right)$ of the crossed-product associated with the restriction of the action to $\Gamma_{1}$, equipped with the induced trace.

Of course, we do not claim that this invariant is complete.

Our treatment considers p.m.p. standard equivalence relations instead of just free p.m.p. group actions. The notion of $L^{2}$-Betti numbers for these objects, introduced in [Gab02a], gives a criterion for free indecomposability:

Theorem 1.14 (Th. 4.18). If $\mathcal{R}$ is a nowhere hyperfinite p.m.p. standard equivalence relation on $(X, \mu)$ with $\beta_{1}(\mathcal{R}, \mu)=0$, then it is freely indecomposable.

Our main result is Theorem 5.1 which describes the kind of uniqueness one can expect in a free product decomposition into $\mathscr{F} \mathcal{I}$ subrelations.

Some parts of our work may be led in the purely Borel theoretic context. For instance, we show that a treeable $\mathscr{F} \mathcal{I}$ equivalence relation is necessarily smooth (Proposition 4.6). The proofs of Theorem 1.5, Corollary 1.9 and Corollary 1.11 are given in Section 6.4.

\section{Free product decompositions}

2.1. Generalities. Let $X$ be a standard Borel space. All the equivalence relations we will consider are Borel with countable classes. By countable, we mean "at most countable". In the measured context, $X$ is equipped with a non-atomic finite measure $\mu$ and the equivalence relations are measure preserving (m.p.) (resp. probability measure preserving (p.m.p.) when $\mu$ is a probability measure) and the following definitions are understood up to a null-set.

Since we are about to consider, on a standard Borel space $X$, equivalence relations that may be defined only on a subset of $X$, we set: 
Definition 2.1. The Borel set on which a countable standard Borel equivalence relation $\mathcal{R}$ is defined will be called its domain and will be denoted by $\mathrm{D}(\mathcal{R})$.

Recall that a complete section is a Borel subset of $\mathrm{D}(\mathcal{R})$ that meets all the classes. A fundamental domain is a Borel subset of $\mathrm{D}(\mathcal{R})$ that meets each class exactly once. An equivalence relation is smooth if it admits a fundamental domain. An equivalence relation is finite if all its classes are finite. In this case, it is smooth. In the probability measure preserving case, $\mathcal{R}$ is smooth if and only if it is finite. $\mathcal{R}$ is aperiodic if its classes are all infinite on $\mathrm{D}(\mathcal{R})$. If $U \subset \mathrm{D}(\mathcal{R})$, we denote by $\mathcal{R} \mid U$ the restriction $\mathcal{R} \cap U \times U$ of $\mathcal{R}$ to $U$, and its domain is $\mathrm{D}(\mathcal{R} \mid U)=U$. The relation $\mathcal{R}$ is trivial if $\mathcal{R}=\{(x, x): x \in \mathrm{D}(\mathcal{R})\}$, i.e. if its classes are reduced to singletons. The equivalence relation $\mathcal{R}$ on $\mathrm{D}(\mathcal{R}) \subset X$ naturally extends to an equivalence relation on the whole of $X$ by setting the class of $x \in X \backslash \mathrm{D}(\mathcal{R})$ to be reduced to the singleton $\{x\}$. We use the same notation $\mathcal{R}$ for the extended relation since it will be clear from the context what we are considering. The full group $[\mathcal{R}]$ of $\mathcal{R}$ is the group of all Borel isomorphisms of $\mathrm{D}(\mathcal{R})$ whose graph is contained in $\mathcal{R}$. The full pseudogroup $[[\mathcal{R}]]$ is the family of all Borel partial isomorphisms between Borel subsets of $\mathrm{D}(\mathcal{R})$ whose graph is contained in $\mathcal{R}$. The equivalence relation $S$ is a subrelation of $\mathcal{R}$ if $\mathrm{D}(\mathcal{S}) \subset \mathrm{D}(\mathcal{R})$ and $(x, y) \in \mathcal{S}$ implies $(x, y) \in \mathcal{R}$. If $\mathcal{S}$ is a subrelation of $\mathcal{R}$ and $\phi: A \rightarrow B$ is a partial isomorphism in the full pseudogroup [[R] of $\mathcal{R}$ whose target $B$ is contained in $\mathrm{D}(S)$ then

$$
\phi^{-1} S \phi
$$

denotes the equivalence relation of domain $A$ defined by $(x, y) \in \phi^{-1} \delta \phi$ if and only if $(\phi(x), \phi(y)) \in S$. It is the image of $\delta \mid B$ under $\phi^{-1}$. Two subrelations $\delta_{1}$ and $\delta_{2}$ of $\mathcal{R}$ are said inner conjugate in $\mathcal{R}$ if there is a partial isomorphism $\phi \in[[\mathcal{R}]]$ with domain $\mathrm{D}\left(S_{2}\right)$ and target $\mathrm{D}\left(S_{1}\right)$ such that $S_{2}=\phi^{-1} \delta_{1} \phi$.

\subsection{Free products}

Definition 2.2 (see [Gab00], Déf. IV.9). A countable family of equivalence relations $\left(\mathcal{R}_{i}\right)_{i \in I}$ with domains $\mathrm{D}\left(\mathcal{R}_{i}\right) \subset X$ is freely independent if the following holds: for any $n$-tuple $\left(x_{1}, \ldots, x_{n}\right)$ of distinct elements of $X$ such that $\left(x_{j}, x_{j+1}\right) \in \mathcal{R}_{i_{j}}$ (for $j=1, \ldots, n$ and $x_{n+1}:=x_{1}$ ), there is an index $j$ such that $i_{j}=i_{j+1}$. The equivalence relation $\mathcal{R}$ is decomposed as the free product

$$
\mathcal{R}=\underset{i \in I}{*} \mathcal{R}_{i}
$$

or is the free product of the countable family $\left(\mathcal{R}_{i}\right)_{i \in I}$ if the family of subrelations is freely independent and generates $\mathcal{R}$ (in particular $\mathrm{D}(\mathcal{R})=\bigcup_{i \in I} \mathrm{D}\left(\mathcal{R}_{i}\right)$ ). The $\mathcal{R}_{i}$ are the factors or the components of the free product decomposition.

Lemma 2.3. Let $\mathcal{R}=\mathcal{R}_{1} * \mathcal{R}_{2} * \mathcal{R}_{3}$ be decomposed as a free product. Consider $S_{1}$ and $S_{2}$ two subrelations of $\mathcal{R}_{1}$ and $\mathcal{R}_{2}$ that are inner conjugate in $\mathcal{R}$, then $S_{1}$ (and $\left.S_{2}\right)$ is smooth. 
Proof. Let $\phi \in[[\mathcal{R}]], \phi: \mathrm{D}\left(S_{2}\right) \rightarrow \mathrm{D}\left(S_{1}\right)$ such that $S_{2}=\phi^{-1} S_{1} \phi$. Assume first that $\phi$ decomposes as a product of partial isomorphisms taken strictly from the [[ $\left.\mathcal{R}_{i}\right]$, i.e. $\phi=\phi_{r_{n}} \ldots \phi_{r_{2}} \phi_{r_{1}}$, such that for each $j: \phi_{r_{j}} \in\left[\left[\mathcal{R}_{k_{j}}\right]\right], k_{j} \neq k_{j+1}$ and for every $z$ in its domain $\phi_{r_{j}}(z) \neq z$. Any $(x, y) \in \mathcal{S}_{2}$ defines, by introducing the right subwords of $\phi$, a "rectangular" cycle

$$
\begin{aligned}
& x \stackrel{\mathcal{R}_{k_{1}}}{\sim} \phi_{r_{1}}(x) \stackrel{\mathcal{R}_{k_{2}}}{\sim} \phi_{r_{2}} \phi_{r_{1}}(x) \sim \ldots \stackrel{\mathcal{R}_{k_{n-1}}}{\sim} \phi_{r_{n-1}} \ldots \phi_{r_{2}} \phi_{r_{1}}(x) \stackrel{\mathcal{R}_{k_{n}}}{\sim} \phi(x) \\
& s_{2} 2 \\
& y \stackrel{\mathcal{R}_{k_{1}}}{\sim} \phi_{r_{1}}(y) \stackrel{\mathcal{R}_{k_{2}}}{\sim} \phi_{r_{2}} \phi_{r_{1}}(y) \sim \ldots \stackrel{\mathcal{R}_{k_{n-1}}}{\sim} \phi_{r_{n-1}} \ldots \phi_{r_{2}} \phi_{r_{1}}(y) \stackrel{\mathcal{R}_{k_{n}}}{\sim} \phi(y)
\end{aligned}
$$

that may be shorten by definition of free products. Due to strictness, the only possible shortenings may occur around the vertical sides: after a possible shortening of the horizontal sides in case $k_{1}=2$ or $k_{n}=1$, the extreme points have to coincide, so that $\delta_{1}$ and $S_{2}$ are trivial. The general case reduces to this after a decomposition of the domain of $\phi$ into pieces where it satisfies the above assumption. Its restrictions to the pieces being trivial, $\delta_{1}$ and $\delta_{2}$ follow smooth.

2.3. Graphings and treeings. Recall from [Lev95], [Gab00] that a countable family of partial isomorphisms $\Phi=\left(\phi_{i}\right)_{i \in I}$ is called a graphing and defines an equivalence relation $\mathcal{R}_{\Phi}=\langle\Phi\rangle=\left\langle\phi_{i}: i \in I\right\rangle$ on $\mathrm{D}\left(\mathcal{R}_{\Phi}\right)=$ the union of the domains and the targets of the $\phi_{i}$ 's. It is a treeing if any equation $\phi_{i_{1}}^{\varepsilon_{1}} \phi_{i_{2}}^{\varepsilon_{2}} \cdots \phi_{i_{n}}^{\varepsilon_{n}}(x)=x$ (with $\left.\varepsilon_{i_{j}}= \pm 1\right)$ implies there is an index $j$ such that $i_{j}=i_{j+1}$ and $\varepsilon_{i_{j}}=-\varepsilon_{i_{j+1}}$. An equivalence relation is treeable if it admits a generating treeing. The notion of treeing was introduced by S. Adams [Ada88] and proved to be very useful in [Gab98] and [Gab00].

We recall some properties of treeable equivalence relations and their connections with free products.

Proposition 2.4. The following holds in the Borel theoretic context:

1. A subrelation of a treeable equivalence relation is itself treeable.

2. If $\Phi=\left(\phi_{i}\right)_{i \in I}$ is a treeing, then $\mathcal{R}_{\Phi}$ is the free product of the subrelations generated by the individual partial isomorphisms $\mathcal{R}_{\Phi}=\mathcal{*}_{i \in I}\left\langle\phi_{i}\right\rangle$.

3. A free product of treeable equivalence relations is treeable.

4. A treeable equivalence relation is freely decomposed as a free product of finite subrelations.

Proof. Item 1 is Theorem IV.4 in [Gab00] (where the proof does not make use of the measure). This has also been shown independently by Jackson-Kechris-Louveau [JKL02]. See also [Alv08a] for a geometric approach. Item 2 is immediate from the definitions (see [Gab00], Ex. IV.10). So is also item 3: a treeing for the free product is made of the union of treeings for the factors. As for item 4, by a result 
of Slaman-Steel and Weiss ([SS88], [Wei84]), each singly generated relation $\left\langle\phi_{i}\right\rangle$ is hyperfinite. We now claim that a hyperfinite equivalence relation is a free product of finite equivalence relations. To that end, it is enough to show that if $\mathcal{R}_{1}$ is a subrelation of a finite equivalence relation $\mathcal{R}_{2}$ then there exists a (finite) subrelation $\mathcal{R}_{1}^{\prime}$ of $\mathcal{R}_{2}$ such that $\mathcal{R}_{2}=\mathcal{R}_{1} * \mathcal{R}_{1}^{\prime}$. Indeed, given fundamental domains $D_{1}$ and $D_{2}$ of $\mathcal{R}_{1}$ and $\mathcal{R}_{2}$, we get a Borel finite-to-one projection $\pi: D_{1} \rightarrow D_{2}$ whose pre-images naturally define the required $\mathcal{R}_{1}^{\prime}$ on $D_{1}$.

An action $\Gamma \curvearrowright(X, \mu)$ is treeable if the equivalence relation it generates is treeable. If $\Gamma$ is measure equivalent with a free group then it admits a treeable p.m.p. free action.

Question 2.5 ([Gab00], Question VI.2). Are there groups with both treeable and non-treeable free p.m.p. actions?

2.4. Slidings and slicings. We now consider two banal ways of freely decomposing an equivalence relation.

Definition 2.6 (Slicing). A slicing of $\mathcal{R}$ is the free product decomposition

$$
\mathcal{R}=\underset{j \in J}{*} \mathcal{R} \mid V_{j}
$$

affiliated with an $\mathcal{R}$-invariant Borel partition $\bigsqcup_{j \in J} V_{j}$ of the domain $\mathrm{D}(\mathcal{R})$.

Definition 2.7 (Sliding). Let $U \subset \mathrm{D}(\mathcal{R})$ be a complete section of $\mathcal{R}$. A sliding of $\mathcal{R}$ to $U$ consists in a smooth subrelation $\mathcal{T}<\mathcal{R}$ defined on $\mathrm{D}(\mathcal{R})$ with fundamental domain $U$ and in the corresponding free product decomposition

$$
\mathcal{R}=\mathcal{R} \mid U * \mathcal{T}
$$

An explicit construction of such a smooth (thus treeable) subrelation $\mathcal{T}$ for each such $U$ may be found in [Gab00], Lem. II.8, where the notion is introduced in a measured context. Notice that the proof does not make use of the measure. From this, one can deduce an easy particular case of Theorem 3.1 from next section:

Proposition 2.8. Assume that $\mathcal{R}=\boldsymbol{*}_{j \in J} \mathcal{R}_{j}$ is a free product decomposition with $\mathrm{D}\left(\mathcal{R}_{j_{0}}\right)=\mathrm{D}(\mathcal{R})$ for some $j_{0}$ and $U$ a complete section for each $\mathcal{R}_{j}$. Then we have $\mathcal{R}\left|U=*_{j \in J} \mathcal{R}_{j}\right| U * \mathcal{T}$, where $\mathcal{T}$ is a treeable subrelation.

Proof. Consider slidings $\mathcal{R}_{j}=\mathcal{R}_{j} \mid U * \mathcal{T}_{j}$ and inject them in the decomposition of $\mathcal{R}=*_{j \in J}\left(\mathcal{R}_{j} \mid U * \mathcal{T}_{j}\right)$. For $j \neq j_{0}$, the sliding $\mathcal{T}_{j} * \mathcal{T}_{j_{0}}=\left(\mathcal{T}_{j} * \mathcal{T}_{j_{0}}\right) \mid U * \widetilde{\mathcal{T}}_{j_{0}}$, gives the global sliding

$$
\underset{j \in J}{*} \mathcal{T}_{j}=\underset{j \in J \backslash\left\{j_{0}\right\}}{*}\left(\mathcal{T}_{j} * \mathcal{T}_{j_{0}}\right) \mid U * \mathcal{T}_{j_{0}} .
$$


It follows that

$$
\mathcal{R}\left|U=\underset{j \in J}{*} \mathcal{R}_{j}\right| U * \overbrace{\underset{\substack{* \\ j \in J \backslash\left\{j_{0}\right\}}}{\mathcal{T}}\left(\mathcal{T}_{j} * \mathcal{T}_{j_{0}}\right) \mid U}
$$

where $\mathcal{T}$ is treeable by Proposition 2.4, items 1 and 3.

\section{Theorems à la Kurosh after [Alv08a]}

We will make a crucial use in our construction of some tools introduced by the first named author, namely the following two analogues of Kurosh's theorem [Kur34] for subgroups of free products, in the context of p.m.p. standard equivalence relations. The first one concerns the particular situation of a subrelation which is simply the restriction to some Borel subset of a given free product.

Theorem 3.1 (A la Kurosh for restrictions [Alv08a]). Let

$$
S=\underset{i \in I}{*} S_{i}
$$

be a free product decomposition of $S$ and $Y \subset X$ a complete section for $S$. Then $S$ admits a refined free product decomposition induced by slicings of the factors $S_{i}$,

$$
S_{i}=\underset{k \in K(i)}{*} \delta_{i} \mid X_{k}, \quad D\left(S_{i}\right)=\coprod_{k \in K(i)} X_{k},
$$

such that the restriction $S \mid Y$ admits a free product decomposition:

$$
\left.S \mid Y=\underset{i \in I}{*} \underset{k \in K(i)}{*} \mathcal{V}_{k}\right) * \mathcal{T}
$$

where $\mathcal{T}$ is a treeable subrelation; and for each $i \in I$ :

1. for each $k \in K(i)$, there is a partial isomorphism $\phi_{k} \in[[S]]$, defined on the domain $\mathrm{D}\left(\mathcal{V}_{k}\right)$, that inner conjugates $\mathcal{V}_{k}$ with $S_{i}$ restricted to the target of $\phi_{k}$ :

$$
\mathcal{V}_{k}=\phi_{k}^{-1} \delta_{i} \phi_{k}
$$

and $X_{k}=S_{i} \phi_{k}\left(\mathrm{D}\left(\mathcal{V}_{k}\right)\right)$ is the $S_{i}$-saturation of the image $\phi_{k}\left(\mathrm{D}\left(\mathcal{V}_{k}\right)\right)$;

2. if $\mathrm{D}\left(S_{i}\right) \cap Y$ is non-empty, then there is $k \in K(i)$ such that $\mathcal{V}_{k}=S_{i} \mid \mathrm{D}\left(S_{i}\right) \cap Y$

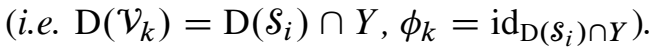

Compare with the analog result [IPP08], Prop. 7.4(2); both the statement and the proof are much less intricate, due to the assumption that the factors $S_{i}$ are ergodic. This Theorem 3.1 is itself of course a little bit more precise than the next one which describes the general situation of a subrelation in a free product. 
Theorem 3.2 (À la Kurosh [Alv08a]). Let

$$
\mathcal{S}=\underset{i \in I}{*} S_{i}
$$

be a free product decomposition of $\mathcal{S}$. If $\mathcal{R}<\mathcal{S}$ is a subrelation of $S$ with non-null domain $\mathrm{D}(\mathcal{R}) \subset X$, then $\mathcal{R}$ admits a free product decomposition

$$
\left.\mathcal{R}=\underset{i \in I}{*} \underset{k \in K(i)}{\underset{*}{*}} \mathcal{V}_{k}\right) * \mathcal{T}
$$

where $\mathcal{T}$ is a treeable subrelation; and for each $i \in I$ :

1. for each $k \in K(i)$, there is a partial isomorphism $\phi_{k} \in[[\delta]]$ defined on the domain $\mathrm{D}\left(\mathcal{V}_{k}\right)$ such that

$$
\mathcal{V}_{k}=\mathcal{R} \cap \phi_{k}^{-1} \delta_{i} \phi_{k}
$$

In particular, $\mathcal{V}_{k}$ is inner conjugate with a subrelation of $S_{i}$.

2. there is $k \in K(i)$ such that $\mathcal{V}_{k}=\mathcal{R} \cap S_{i}$ (when this intersection is not trivial), $\mathrm{D}\left(\mathcal{V}_{k}\right)=\mathrm{D}(\mathcal{R}) \cap \mathrm{D}\left(S_{i}\right)$ and $\phi_{k}=i d_{\mathrm{D}\left(\mathcal{V}_{k}\right)}$.

Remark 3.3. It does not matter whether $\mathrm{D}(\mathcal{R})$ is a complete section of $\delta$ or not.

Remark 3.4. If one of the original factors, say $S_{i_{0}}$, is treeable, then the factors $\mathcal{V}_{k}$ associated with $k \in K\left(i_{0}\right)$ in Theorem 3.1 or Theorem 3.2 follow treeable so that $*_{k \in K\left(i_{0}\right)} \mathcal{V}_{k} * \mathcal{T}$ is treeable (see Proposition 2.4). More generally, if the $\mathcal{V}_{k}$ in a certain collection are treeable, one may assemble them together with $\mathcal{T}$ to form a treeable relation that may be put in place of $\mathcal{T}$, in the above theorems.

\section{Freely indecomposable equivalence relations}

Observing that slicings (Definition 2.6) and slidings (Definition 2.7) decompose an equivalence relation as a free product in a somewhat trivial way leads to set the following definition.

\subsection{Free indecomposability}

Definition 4.1 (Inessential free product decomposition). A free product decomposition $\mathcal{R}=*_{j \in J} \mathcal{R}_{j}$, of a (countable) standard Borel equivalence relation on $X$ is called inessential if there is a Borel set $U \subset X$ such that:

1. $U$ admits a Borel $\mathcal{R} \mid U$-invariant partition $U=\bigsqcup_{j \in J} U_{j}$;

2. for each $j \in J, \mathcal{R}\left|U_{j}=\mathcal{R}_{j}\right| U_{j}$;

3. $U$ is a complete section for $\mathcal{R}$. 
We then say that the partition is a trivialization of the decomposition.

Remark 4.2. In the measured context, all identities are understood up to a set of measure zero.

Remark 4.3. If the decomposition $\mathcal{R}=\mathcal{*}_{j \in J} \mathcal{R}_{j}$ is trivialized by $U=\bigsqcup_{j \in J} U_{j}$ :

1. It induces the slicing $\mathcal{R}\left|U=\boldsymbol{*}_{j \in J} \mathcal{R}_{j}\right| U_{j}=\boldsymbol{*}_{j \in J} \mathcal{R} \mid U_{j}$, and $U$ being a complete section, a sliding/slicing decomposition $\mathcal{R}=*_{j \in J} \mathcal{R}_{j} \mid U_{j} * \mathcal{T}$, where $\mathcal{T}$ is a smooth treeable equivalence relation with fundamental domain $U$.

2. We insist that the $\mathcal{R} \mid U$-invariance of the partition means that the $\mathcal{R}$-saturations $V_{j}:=\mathcal{R} U_{j}$ of the $U_{j}$ have trivial mutual intersections and partition $X$, leading to the slicing:

$$
\mathcal{R}=\underset{j \in J}{*} \mathcal{R} \mid V_{j}
$$

Proposition 4.4. Assume the free product decomposition $\mathcal{R}=\boldsymbol{*}_{j \in J} \mathcal{R}_{j}$ is trivialized by $U=\bigsqcup_{j \in J} U_{j}$. Then,

1. $\bar{U}=\bigsqcup_{j \in J} \bar{U}_{j}$, where $\bar{U}_{j}:=\mathcal{R}_{j} U_{j}$ is the $\mathcal{R}_{j}$-saturation of $U_{j}$, also trivializes the free product decomposition.

2. If $j \in J$

- the subrelation $\mathcal{R}_{j}$ is trivial when restricted to $\bar{U}_{i}$, for $i \neq j$;

- the subrelation $\mathcal{R}_{j}$ is smooth when restricted to $X \backslash \bar{U}_{j}$.

3. If $\mathcal{R}$ is measure preserving, $\mathrm{D}\left(\mathcal{R}_{i}\right)=\mathrm{D}(\mathcal{R})$ and $\mathcal{R}_{i}$ is aperiodic, then $X=V_{i}$ and $\mathcal{R}=\mathcal{R}_{i}$ almost everywhere.

4. If $\mathcal{R}$ is ergodic, $U$ equals one of the $U_{j}$ 's, say $U_{j_{0}}$, and $\mathcal{R}=\mathcal{R}_{j_{0}} \mid U * \mathcal{T}$, where $\mathcal{T}$ is a smooth treeing admitting $U$ as fundamental domain.

Proof. The only non-obvious facts are (possibly) items 1 and 2:

1. If $\bar{x}, \bar{y} \in \bar{U}_{j}$ are $\mathcal{R}$-equivalent, there are $\mathcal{R}$-equivalent points $x, y \in U_{j}$ such that $x \mathcal{R}_{j} \bar{x}$ and $\bar{y} \mathcal{R}_{j} y$. By $\mathcal{R}\left|U_{j}=\mathcal{R}_{j}\right| U_{j}, \bar{x}, \bar{y}$ follow $\mathcal{R}_{j}$-equivalent.

2. The first part is clear. As for the second part, it is then enough to show (since $X=\bigcup_{j} V_{j}$ ) that $\mathcal{R}_{j}$ restricted to $V_{i} \backslash \bar{U}_{i}=\mathcal{R} U_{i} \backslash \mathcal{R}_{i} U_{i}$ is smooth for every $i \in I$. The $\mathcal{R}_{i}$-slicing affiliated with $V_{i}=\bar{U}_{i} \amalg V_{i} \backslash \bar{U}_{i}$ and the $\mathcal{R}$-invariance of $V_{i}$ lead to the free product decomposition $\mathcal{R}\left|V_{i}=\mathcal{R}_{i}\right| \bar{U}_{i} * \mathcal{R}_{i} \mid\left(V_{i} \backslash \bar{U}_{i}\right) *\left(\mathcal{*}_{j \in J \backslash\{i\}} \mathcal{R}_{j} \mid V_{i}\right)$. Any partial isomorphism $\phi \in[[\mathcal{R}]]$ with domain $\subset \bar{U}_{i}$ and target $\subset V_{i} \backslash \bar{U}_{i}$ inner conjugates the restriction of each of the other factors with a subrelation of $\mathcal{R}\left|\bar{U}_{i}=\mathcal{R}_{i}\right| \bar{U}_{i}$. By Lemma 2.3, these subrelations are smooth. Since $\bar{U}_{i}$ is a complete section for $\mathcal{R} \mid V_{i}$, there are enough such $\phi$, and the conclusion follows.

Definition 4.5 ( $\mathcal{F} \mathcal{I}$ equivalence relation). A countable standard Borel equivalence relation $\mathcal{R}$ is freely indecomposable $(\mathcal{F} \mathcal{I}$ ) if any free product decomposition $\mathcal{R}=$ $\mathcal{R}_{1} * \mathcal{R}_{2} * \cdots * \mathcal{R}_{i} * \cdots$ is inessential in the sense of Definition 4.1. 
For instance, any finite equivalence relation is $\mathcal{F} \mathcal{I}$. See Remark 4.2 in the measured context.

Proposition 4.6. If $\mathcal{R}$ is treeable (for instance hyperfinite) and $\mathcal{F} \mathcal{I}$ then it is smooth. In fact, any p.m.p. aperiodic treeable equivalence relation admits an essential decomposition in two pieces $\mathcal{R}=\mathcal{R}_{1} * \mathcal{R}_{2}$.

By Ornstein-Weiss theorem [OW80], Proposition 4.6 entails:

Corollary 4.7. Every free p.m.p. action of an infinite amenable group is non- $\mathcal{F} \mathcal{I}$.

Proof of Proposition 4.6. From Proposition 2.4, $\mathcal{R}$ decomposes as a free product $*_{i \in I} \mathcal{R}_{i}$ of finite subrelations. The property $\mathcal{R}\left|U_{i}=\mathcal{R}_{i}\right| U_{i}$ in Definition 4.1 (2) implies that $\mathcal{R}$ is smooth. In the p.m.p. context, the $X$ splits in two $\mathcal{R}$-invariant Borel subsets $X_{\infty} \coprod X_{h}$ where the classes have infinitely many ends (resp. where $\mathcal{R}$ is hyperfinite) [Ada90]. On $X_{h}, \mathcal{R}$ is generated by a free action of $\mathbb{Z} / 2 \mathbb{Z} * \mathbb{Z} / 2 \mathbb{Z}$. On $X_{\infty}$, we claim that one can find a treeing $\Phi=\left(\varphi_{1}\right) \vee \Phi_{2}$ of $\mathcal{R}$ such that the domain $A_{1}$ of $\varphi_{1}$ is non-negligible and $\Phi_{2}$ generates a subrelation $\mathcal{R}_{2}$ on $X_{\infty}$ that is aperiodic. Assuming this claim, the free product decomposition $\mathcal{R} \mid X_{\infty}=\left\langle\varphi_{1}\right\rangle * \mathcal{R}_{2}$ is essential: Proposition 4.4(2) with aperiodicity of $\mathcal{R}_{2}$ implies $\bar{U}_{2}=X_{\infty}$ (a.e.) for any hypothetical trivialization. But again by Proposition $4.4(2),\left\langle\varphi_{1}\right\rangle$ should be trivial on $\bar{U}_{2}=X_{\infty}$.

Let us prove the claim. Indeed, we show that: if $\Phi$ is a treeing of $\mathcal{R}$ such that the associated graphs $\Phi[x]$ (see [Gab00]) for all $x \in X$ have infinitely many ends ${ }^{1}$, then up to a subdivision (i.e. the splitting of a generator in two generators by a partition of its domain in two parts) one can pick one of the generators $\varphi_{1} \in \Phi$ whose removal produces a graphing $\Phi_{2}:=\Phi \backslash\left(\varphi_{1}\right)$ which generates an aperiodic subrelation.

If for every $x \in X$, its valency in $\Phi[x]$ is $\geq 3$, then pick any $\varphi \in \Phi$ and choose a non-negligible Borel subset $A$ of its domain such that $A \cap \varphi(A)=\emptyset$. Then $\varphi_{1}:=\varphi \mid A$ and $\phi_{2}$ obtained by replacing $\varphi$ by its restriction to $X \backslash A$ will do (in the graphs $\Phi_{2}[x]$ the valency of every vertex is $\geq 2$ ).

In general, let $X_{\text {core }}$ be the set of $x \in X$ such that (when seen as a vertex in $\Phi[x]) x$ belongs to the core of $\Phi[x]$ (i.e. the union of the geodesic lines). Let $\Phi_{\text {core }}$ be the graphing made of the restrictions $\tilde{\varphi}$ of $\varphi \in \Phi$ to the subset of their domain where $x$ and $\varphi(x)$ both belong to $X_{\text {core }}$. By assumption, $X_{\text {core }}$ is a complete section for $\mathcal{R}$. Moreover $\mathcal{R}_{\Phi_{\text {core }}}=\mathcal{R} \mid X_{\text {core }}$ and $\Phi_{\text {core }}$ is a treeing on $X_{\text {core }}$ whose graphs $\Phi_{\text {core }}[x]$ have infinitely many ends and no leaves. Let $X_{\geq 3} \subset X_{\text {core }}$ be the set of $x$ whose valency in $\Phi_{\text {core }}[x]$ is $\geq 3$. It is again a complete section for $\mathcal{R}$. Pick a partial isometry $\tilde{\varphi} \in \Phi_{\text {core }}$ whose domain intersects $X_{\geq 3}$ in a non-null set (up to replacing $\tilde{\varphi}$ by $\tilde{\varphi}^{-1}$ ). Then choose a non-negligible subset $A$ (in this intersection) such that $A \cap f(A)=\emptyset$, where $f(x)$ is the first point in $X_{\geq 3}$ along a ray issuing from $x$ in the direction of $\tilde{\varphi}(x)$ in $\Phi_{\text {core }}[x]$ (again, vertices in $\Phi_{\text {core }}[x]$ and points in $X_{\text {core }}$ are

\footnotetext{
${ }^{1}$ I.e., there are infinitely many different rays issuing from any vertex.
} 
identified). Set $\varphi_{1}:=\tilde{\varphi} \mid A$. By construction, for every point $x \in X_{\geq 3}$, its long star (the rays in $\Phi_{\text {core }}[x]$ issuing from $x$ until reaching another point in $X_{\geq 3}$ ) meets at most one edge associated with $\varphi_{1}$. Thus, removing $\varphi_{1}$ from $\Phi_{\text {core }}($ or $\Phi)$ produces a graphing with infinite orbits.

Proposition 4.8. For p.m.p. standard aperiodic equivalence relations, the Definition 4.5 can be stated equivalently for free product decompositions in two pieces.

Proof. Let $\mathcal{R}$ be a standard aperiodic p.m.p. equivalence relation. Assuming that any free product decomposition into two pieces $\mathcal{R}=\delta_{1} * \delta_{2}$ is inessential, we show that any free product decomposition $\mathcal{R}=*_{j \in J} \mathcal{R}_{j}$ into at most countably many pieces is also inessential.

For each $i \in J$, let $\mathcal{R}_{i}^{\prime}=*_{j \in J \backslash\{i\}} \mathcal{R}_{j}$ and consider a trivialization $U_{i} \amalg U_{i}^{\prime}$ associated with the free product decomposition, $\mathcal{R}=\mathcal{R}_{i} * \mathcal{R}_{i}^{\prime}$. Moreover $U_{i}$ may be assumed $\mathcal{R}_{i}$-saturated (Proposition 4.4 (1)). We claim that $V=\bigsqcup U_{i}$ trivializes the free product decomposition $*_{j \in J} \mathcal{R}_{j}$. Since $\mathcal{R}_{i}\left|U_{i}=\mathcal{R}\right| U_{i}$ is aperiodic (when $U_{i}$ is non negligible) and $\mathcal{R}_{i} \mid X \backslash U_{i}$ is smooth (Proposition 4.4 (2)), the only point to check is that $V$ is a complete section. The complement of its saturation $Y=X \backslash \mathscr{R} V$ is contained in $\cap_{i \in J}\left(X \backslash U_{i}\right)$, so that $\mathcal{R}\left|Y=*_{j \in J} \mathcal{R}_{j}\right| Y$ is treeable.

Under a p.m.p. assumption, Proposition 4.6 and $\mu(Y) \neq 0$ would produce an essential free product decomposition in two pieces of $\mathcal{R} \mid Y$ (thus also of $\mathcal{R}$ ), leading to a contradiction. Thus $Y$ is negligible.

\subsection{Properties of free indecomposability}

Remark 4.9. Given an $\mathcal{R}$-invariant partition $\mathrm{D}(\mathcal{R})=Y \amalg Z$, then $\mathcal{R}$ is $\mathcal{F} \mathcal{I}$ if and only if $\mathcal{R} \mid Y$ and $\mathcal{R} \mid Z$ are $\mathcal{F} \mathcal{I}$. In particular, if $\mathcal{R}$ is $\mathscr{F} \mathcal{I}$, then the extension of $\mathcal{R}$ to $X$ (by trivial classes outside $\mathrm{D}(\mathcal{R})$ ) is also $\mathcal{F} \mathcal{I}$.

Proposition 4.10. If $\mathcal{R}$ is $\mathcal{F} \mathcal{I}$, then for every non-null Borel set $Y$, the restriction $\mathcal{R} \mid Y$ is also $\mathcal{F} \mathcal{I}$.

Proof. By Remark 4.9, one may assume that $Y$ is a complete section of $\mathcal{R}$ and that $\mathcal{R}$ is aperiodic. Any free product decomposition $\mathcal{R} \mid Y=\mathcal{*}_{i \in I} \mathcal{R}_{i}$ leads by sliding to $\mathcal{R}=\mathcal{*}_{i \in I} \mathcal{R}_{i} * \mathcal{T}$ where $\mathcal{T}$ is a treeing with fundamental domain $Y$. The $\mathcal{F} \mathcal{I}$ property for $\mathcal{R}$ gives a trivialization $\bigsqcup_{i \in I} U_{i} \amalg U_{\mathcal{T}}$, such that $U_{i} \subset \mathrm{D}\left(\mathcal{R}_{i}\right) \subset Y$, $\mathcal{R}\left|U_{i}=\mathcal{R}_{i}\right| U_{i}$ and $\mathcal{R}\left|U_{\mathcal{T}}=\mathcal{T}\right| U_{\mathcal{T}}$. Since $\mathcal{T}$ is smooth, $U_{\mathcal{T}}$ is negligible (by aperiodicity). It follows that $\bigsqcup_{i \in I} U_{i} \subset Y$ gives a trivialization for the restriction of $\mathcal{R} \mid Y$.

Proposition 4.11 (Stable orbit equivalence invariance). If $\mathcal{R}$ and $\mathcal{S}$ are stably orbit equivalent, then $\mathcal{R}$ is $\mathcal{F} \mathcal{I}$ if and only if $S$ is $\mathcal{F} \mathcal{I}$. 
Proof. The statement being clear for orbit equivalence, it remains to show: if $Y$ a complete section of $\mathcal{R}$ and $\mathcal{R} \mid Y$ is $\mathcal{F} \mathcal{I}$, then $\mathcal{R}$ is also $\mathcal{F} \mathcal{I}$. One may assume that $\mathcal{R}$ is aperiodic. For each free product decomposition $\mathcal{R}=\mathcal{*}_{i \in I} \mathcal{R}_{i}$, Theorem 3.1 delivers a free product decomposition of the restriction $\mathcal{R} \mid Y=*_{i \in I}\left(*_{k \in K(i)} \phi_{k}^{-1} \mathcal{R}_{i} \phi_{k}\right) *$ $\mathcal{T}$, with $\mathcal{T}$ treeable. The $\mathcal{F} \mathcal{I}$-property for $\mathcal{R} \mid Y$ gives a trivialization

$$
\coprod_{i \in I}\left(\coprod_{k \in K(i)} U_{k}\right) \coprod U_{\mathcal{T}},
$$

where in particular $(\mathcal{R} \mid Y)\left|U_{\mathcal{T}}=\mathcal{T}\right| U_{\mathcal{T}}$ is treeable (Proposition 2.4 item 1) and thus smooth (by Propositions 4.6 and 4.10). It follows that $U_{\mathcal{T}}$ is negligible (by aperiodicity), and from $(\mathcal{R} \mid Y)\left|U_{k}=\left(\phi_{k}^{-1} \mathcal{R}_{i} \phi_{k}\right)\right| U_{k}=\phi_{k}^{-1} \mathcal{R}_{i} \mid \phi_{k}\left(U_{k}\right) \phi_{k}$ that $\coprod_{i \in I}\left(\bigsqcup_{k \in K(i)} \phi_{k}\left(U_{k}\right)\right)$ trivializes the original decomposition.

Definition 4.12. A countable group is called measurably freely indecomposable $(\mathcal{M} \mathcal{F} \mathcal{I})$ if all its free p.m.p. actions are freely indecomposable $(\widetilde{F} \mathcal{I})$.

Proposition 4.13. Being measurably freely indecomposable is a measure equivalence invariant: if $\Gamma \stackrel{\mathrm{ME}}{\sim} \Lambda$ then $\Gamma$ is $\mathcal{M} \mathcal{F} \mathcal{I}$ iff $\Lambda$ is $\mathcal{M F} \mathcal{F}$.

For instance, such groups as the fundamental group of a closed orientable surface of genus $\geq 2$ are freely indecomposable in the classical sense (they have only one end) but are not $\mathcal{M} \mathcal{F} \mathcal{I}$ since being ME with a non-cyclic free group.

Proof of Proposition 4.13. Consider two standard p.m.p. equivalence relations $\widetilde{\mathcal{R}}$ on $(\tilde{X}, \tilde{\mu})$ and $\mathcal{R}$ on $(X, \mu)$. Let $p: \tilde{X} \rightarrow X$ be a measurable map such that $p_{*}(\tilde{\mu}) \sim \mu$ and $p$ induces, for (almost) every $\tilde{x} \in \tilde{X}$, a bijection between the $\widetilde{R}$-class of $\tilde{x}$ and the $\mathcal{R}$-class of $p(\tilde{x})$. Such a $p$ is a locally bijective morphism from $\widetilde{\mathcal{R}}$ to $\mathcal{R}$.

Remark 4.14. This notion were introduced in [Gab05], p. 1815, as locally one-to-one and onto morphism from $\widetilde{\mathcal{R}}$ to $\mathcal{R}$, and we take this opportunity to correct a regrettable translation mistake that led to use the words one-to-one instead of bijective, all along the paper.

Lemma 4.15. If $\widetilde{R}$ is $\mathcal{F} \mathcal{I}$ then $\mathcal{R}$ is also $\mathcal{F} \mathcal{I}$.

Proof of the lemma. Observe that for a Borel subset $\widetilde{W} \subset \tilde{X}, \widetilde{\mathcal{R}} \mid \widetilde{W}$ is smooth if and only if $\mathcal{R} \mid p(\widetilde{W})$ is smooth (the restrictions are smooth iff their saturations are smooth, iff their classes are finite). Let $\mathcal{R}=*_{j \in J} \mathcal{R}_{j}$ be a free product decomposition of $\mathcal{R}$. It induces via $p$ a free product decomposition accordingly $\widetilde{\mathcal{R}}=*_{j \in J} \widetilde{\mathcal{R}}_{j}$, where $p$ becomes a locally bijective morphism from $\widetilde{\mathcal{R}}_{j}$ to $\mathcal{R}_{j}$ (see [Gab05]). Let $\bigsqcup_{j \in J} \tilde{U}_{j}$ be a trivializing partition of this decomposition such that the $\tilde{U}_{j}$ are $\widetilde{\mathcal{R}}_{j}$ saturated (Proposition 4.4 item 1) and let $U_{j}=p\left(\tilde{U}_{j}\right)$. If $\widetilde{R}_{j}$ is smooth when restricted to 
some Borel subset $\widetilde{Z}_{j} \subset \widetilde{U}_{j}$, then $\widetilde{\mathcal{R}}$ follows smooth on $\widetilde{\mathcal{R}} \widetilde{Z}_{j}$ (by $\widetilde{\mathcal{R}}_{j}\left|\widetilde{U}_{j}=\widetilde{\mathcal{R}}\right| \widetilde{U}_{j}$ ) and the same holds for $\mathcal{R}$ on $p\left(\widetilde{\mathcal{R}} \widetilde{Z}_{j}\right)$, so that $\mathcal{R}$ is $\mathscr{F} \mathcal{I}$ on this saturated part. One thus may assume that the $\widetilde{\mathcal{R}}_{j} \mid \widetilde{U}_{j}$-classes are all infinite. By Proposition 4.4 item $2, \widetilde{\mathcal{R}}_{j}$ is smooth outside $\widetilde{U}_{j}$, so that $p\left(\tilde{X} \backslash \widetilde{U}_{j}\right) \cap p\left(\widetilde{U}_{j}\right)$ is negligible and $\widetilde{U}_{j}=p^{-1} p\left(\widetilde{U}_{j}\right)$. It follows that $\amalg p\left(\widetilde{U}_{j}\right)$ is a trivializing partition for $\mathcal{R}=*_{j \in J} \mathcal{R}_{j}$.

From this lemma, one gets that if $\Gamma \stackrel{\mathrm{ME}}{\sim} \Lambda$ and if $\Gamma$ is not $\mathcal{M F} \mathcal{F}$ (i.e. $\Gamma$ admits some non $\mathscr{F} \mathcal{I}$ p.m.p. free action $\left.\Gamma \curvearrowright^{\alpha}(X, \mu)\right)$ then there is a p.m.p. free action of $\Gamma$ that is both non- $\mathcal{F} \mathcal{I}$ and SOE with a p.m.p. free action of $\Lambda$. Let $(\Omega, \nu)$ be a measure equivalence coupling between $\Gamma$ and $\Lambda$. Consider the coupling $(\Omega \times X, v \times \mu)$, with the diagonal actions induced from $\Gamma \curvearrowright^{\alpha}(X, \mu)$ and the trivial action of $\Lambda$ on $X$. The quotient actions $\Gamma \curvearrowright(\Omega \times X) / \Lambda$ and $\Lambda \curvearrowright \Gamma \backslash(\Omega \times X)$ are free (see [Gab02b]), SOE and the first one is non- $\mathcal{F} \mathcal{I}$ by the above lemma, since it factors onto $\Gamma \curvearrowright^{\alpha}(X, \mu)$. The conclusion of Proposition 4.13 then follows by SOE invariance (Proposition 4.11).

Question 4.16. Are there groups that admit some $\mathcal{F} \mathcal{I}$ and some non-F्F free p.m.p. actions?

4.3. $L^{2}$-Betti numbers. We now consider finite-measure preserving equivalence relations.

Definition 4.17. A measure preserving standard equivalence relation $\mathcal{R}$ on $(X, \mu)$ is called nowhere hyperfinite if for every non-null Borel subset $V \subset X$, the restriction $\mathcal{R} \mid V$ is not hyperfinite.

We establish a criterion for equivalence relations to be $\mathcal{F} \mathcal{I}$. The notion of $L^{2}$ Betti numbers is introduced in [Gab02a]. Some useful properties are recalled in Section 6.1.

Theorem 4.18. If $\mathcal{R}$ is a nowhere hyperfinite finite-measure preserving standard equivalence relation on $(X, \mu)$ with $\beta_{1}(\mathcal{R})=0$, then it is freely indecomposable.

In case $\mathrm{D}(\mathcal{R}) \neq X$ and $\beta_{1}(\mathcal{R})=0$ (of course computed with respect to the restriction of the measure to $\mathrm{D}(\mathcal{R})$ - see Section 6.1), then the extension of $\mathcal{R}$ to $X$ (by trivial classes outside $\mathrm{D}(\mathcal{R})$ ) is also $\mathscr{F} \mathcal{I}$ (see Remark 4.9).

We will prove (Section 6.2 ) more precisely:

Theorem 4.19. Assume $\mathcal{R}$ is an aperiodic finite measure preserving standard equivalence relation with $\beta_{1}(\mathcal{R})=0$ and assume that $\mathcal{R}$ decomposes as a free product $\mathcal{R}=\mathcal{R}_{1} * \mathcal{R}_{2} * \cdots * \mathcal{R}_{i} * \cdots$. Let $U_{i} \subset X$ be the union of the infinite $\mathcal{R}_{i}$-classes, for $i=1,2, \ldots$. Then

1. the mutual intersections are trivial, $\mu\left(U_{i} \cap U_{j}\right)=0$ for $i \neq j$; 
2. the partition $U=U_{1} \bigsqcup U_{2} \bigsqcup \cdots \bigsqcup U_{i} \bigsqcup \cdots$ is $\mathcal{R} \mid U$-invariant;

3. the restrictions $\mathcal{R}_{i} \mid U_{j}$ are trivial for $i \neq j$;

4. $\mathcal{R}\left|U_{i}=\mathcal{R}_{i}\right| U_{i}$ for each $i$;

5. the restriction of $\mathcal{R}$ to the complement $W$ of the saturation $\mathcal{R} U$ is hyperfinite (if $\mu(W)>0$ ).

And thus, if $\mathcal{R}$ is nowhere hyperfinite then the partition of $U$ trivializes the decomposition.

Given the coincidence [Gab02a] of the first $\ell^{2}$-Betti number $\beta_{1}(\Gamma)$ of any countable group $\Gamma$ with the first $L^{2}$-Betti number $\beta_{1}\left(\mathcal{R}_{\alpha}, \mu\right)$ of the orbit equivalence relation defined by any free p.m.p. action $\Gamma \curvearrowright^{\alpha}(X, \mu)$, and since non-amenability implies nowhere hyperfinite, we immediately get:

Corollary 4.20. Every non-amenable countable group $\Gamma$ with vanishing first $\ell^{2}-$ Betti number $\beta_{1}(\Gamma)=0$ is measurably freely indecomposable.

Problem 4.21. Produce examples of $\mathcal{M} \mathscr{F} \mathcal{I}$ groups with $\beta_{1}>0$.

Let us say that a p.m.p. countable standard equivalence relation is accessible if it admits a free product decomposition $\mathcal{R}=*_{j \in J} \mathcal{R}_{j}$ into freely indecomposable subrelations.

Problem 4.22. Find/characterize p.m.p. countable standard equivalence relations, with finite $\beta_{1}$, that are non-accessible.

\section{Bass-Serre rigidity}

Suppose that $\Theta: G_{1} * G_{2} \rightarrow G_{1}^{\prime} * G_{2}^{\prime}$ is an isomorphism of groups where $G_{i}$ and $G_{j}^{\prime}$ are freely indecomposable groups different from $\mathbb{Z}$. Since $\Theta\left(G_{1}\right)$ is a subgroup of $G_{1}^{\prime} *$ $G_{2}^{\prime}$, Kurosh's theorem implies that $\Theta\left(G_{1}\right)$ is a subgroup of a conjugate of $G_{1}^{\prime}$ or $G_{2}^{\prime}$. Up to a permutation of the indices, we assume that $\Theta\left(G_{1}\right)$ is a subgroup of a conjugate $\operatorname{conj}\left(G_{1}^{\prime}\right)$ of $G_{1}^{\prime}$. Another use of Kurosh's theorem implies that $\Theta^{-1}\left(\operatorname{conj}\left(G_{1}^{\prime}\right)\right)$ is a subgroup of a conjugate of $G_{1}$ or $G_{2}$. But since $\Theta^{-1}\left(\operatorname{conj}\left(G_{1}^{\prime}\right)\right)$ contains $G_{1}$, we deduce equality with $G_{1}$, i.e. $\Theta\left(G_{1}\right)=\operatorname{conj}\left(G_{1}^{\prime}\right)$ and in the same way that $\Theta\left(G_{2}\right)=\operatorname{conj}\left(G_{2}^{\prime}\right)$. This observation is the starting point of our main theorem:

Theorem 5.1. Let $\mathcal{R}=*_{p \in P} \mathcal{R}_{p} * \mathcal{T}$ and $\mathcal{R}^{\prime}=*_{p^{\prime} \in P^{\prime}} \mathcal{R}_{p^{\prime}}^{\prime} * \mathcal{T}^{\prime}$ be p.m.p. standard equivalence relations decomposed into free products, where each factor $\mathcal{R}_{p}$ and $\mathcal{R}_{p^{\prime}}^{\prime}$ is freely indecomposable and aperiodic on its domain; and where $\mathcal{T}$ and $\mathcal{T}^{\prime}$ are treeable. If $\mathcal{R}$ and $\mathcal{R}^{\prime}$ are $S O E$, via an isomorphism $\Theta: V \subset \mathrm{D}(\mathcal{R}) \rightarrow V^{\prime} \subset \mathrm{D}\left(\mathcal{R}^{\prime}\right)$ 
then $\mathcal{R}$ and $\mathcal{R}^{\prime}$ admit free product decompositions induced by countable slicings of the factors:

$$
\begin{aligned}
& \forall p \in P, \mathrm{D}\left(\mathcal{R}_{p}\right)=\coprod_{k \in K(p)} X_{k} \text { and } \forall p^{\prime} \in P^{\prime}, \mathrm{D}\left(\mathcal{R}_{p^{\prime}}^{\prime}\right)=\coprod_{k^{\prime} \in K^{\prime}\left(p^{\prime}\right)} X_{k^{\prime}}^{\prime} \\
& \mathcal{R}=\underset{p \in P}{\left.\underset{k}{*} \underset{k \in K(p)}{*} \mathcal{R}_{p} \mid X_{k}\right) * \mathcal{T}} \text { and } \mathcal{R}^{\prime}=\underset{p^{\prime} \in P^{\prime}}{\boldsymbol{*}} \underset{k^{\prime} \in K^{\prime}\left(p^{\prime}\right)}{\left(\underset{R^{\prime}}{*} \mid X_{k^{\prime}}^{\prime}\right) * \mathcal{T}^{\prime}}
\end{aligned}
$$

for which there exists a bijection $\theta: \bigsqcup_{p \in P} K(p) \rightarrow \bigsqcup_{p^{\prime} \in P^{\prime}} K^{\prime}\left(p^{\prime}\right)$ between the index sets such that, denoting $\delta_{k}:=\mathcal{R}_{p} \mid X_{k}$ and $\mathcal{S}_{k^{\prime}}^{\prime}:=\mathcal{R}_{p^{\prime}}^{\prime} \mid X_{k^{\prime}}^{\prime}$, for each $k \in \bigsqcup_{p \in P} K(p)$, the slices $S_{k}$ and $S_{\theta(k)}^{\prime}$ are SOE via an isomorphism between subsets of the domains $\mathrm{D}\left(S_{k}\right)=X_{k}$ and $\mathrm{D}\left(S_{\theta(k)}^{\prime}\right)=X_{\theta(k)}^{\prime}$ of the shape $f^{\prime} \Theta f$, where $f \in[[\mathcal{R}]]$ and $f^{\prime} \in\left[\left[\mathcal{R}^{\prime}\right]\right]$.

The proof of the theorem will be given in Section 6.3.

Remark 5.2. Recall that in case $\mathcal{R}_{p}$ is ergodic, then it admits no non-trivial slicing. If all the $\mathcal{R}_{p}$ and $\mathcal{R}_{p^{\prime}}^{\prime}$ are ergodic, then Theorem 5.1 establishes a bijection between the $\mathcal{R}_{p}$ and the $\mathcal{R}_{p^{\prime}}^{\prime}\left(\theta\right.$ becomes a bijection between the sets of indices $P$ and $\left.P^{\prime}\right)$.

Remark 5.3. In the context of Corollary 1.4, we argue by contraposition: if $\mathcal{R}_{\alpha \mid \Gamma_{2}}$ is ergodic, then a slicing of $\mathcal{R}_{\alpha}$ delivered by Theorem 5.1 contains only one $\Gamma_{2}$-piece. On the other hand, $\mathcal{R}_{\beta}$ has already at least two $\Gamma_{2}$-pieces. Thus, the bijection $\Theta$ of the theorem has to entail an SOE between a $\Gamma_{1}$-slice of $\mathcal{R}_{\alpha}$ and a $\Gamma_{2}$-slice of $\mathcal{R}_{\beta}$.

\section{Proofs}

6.1. Preliminaries. We list some properties of $L^{2}$-Betti numbers of equivalence relations (see [Gab02a]) on the non atomic probability measure space $(X, \mu)$. Recall that the $L^{2}$-Betti numbers are defined with respect to an invariant probability measure [Gab02a]. In case a finite measure is invariant, one usually normalizes it. Thus $\beta_{q}(\mathcal{R})$ stands for the $q$-th $\ell^{2}$-Betti number of $\mathcal{R}$ on $\mathrm{D}(\mathcal{R})$ with respect to the normalized probability measure $\frac{\mu \mid \mathrm{D}(\mathcal{R})}{\mu(\mathrm{D}(\mathcal{R}))}$. If $\mathrm{D}(\mathcal{R}) \subsetneq X$, the notation $\beta_{q}(\mathcal{R}, \mu)$ means that we extend $\mathcal{R}$ trivially outside $\mathrm{D}(\mathcal{R})$ to compute $L^{2}$-Betti numbers according to the probability measure $\mu$.

Proposition 6.1. The following holds:

1. $\beta_{0}(\mathcal{R})=\int_{X} \frac{d \mu(x)}{\# \mathcal{R}(x)}$, the mean value of the inverse of the cardinal of the class of $x$, with the convention $\frac{1}{\infty}=0$. It follows that $\beta_{0}(\mathcal{R}) \in[0,1]$.

2. The relation $\mathcal{R}$ is trivial if and only if $\beta_{0}(\mathcal{R})=1$.

3. $\mathcal{R}$ is aperiodic on $\mathrm{D}(\mathcal{R})$ if and only if $\beta_{0}(\mathcal{R})=0$. 
4. If $\beta_{1}(\mathcal{R})=0$ and $V \subset X$ satisfies $\mu(V)>0$, then $\beta_{1}(\mathcal{R} \mid V)=0$.

5. For a free product: $\beta_{1}\left(\mathcal{R}_{1} * \mathcal{R}_{2}, \mu\right)-\beta_{0}\left(\mathcal{R}_{1} * \mathcal{R}_{2}, \mu\right)=\beta_{1}\left(\mathcal{R}_{1}, \mu\right)-$ $\beta_{0}\left(\mathcal{R}_{1}, \mu\right)+\beta_{1}\left(\mathcal{R}_{2}, \mu\right)-\beta_{0}\left(\mathcal{R}_{2}, \mu\right)+1$.

Proof. We use the notation of [Gab02a]. If the space $(X, \mu)$ admits an $\mathcal{R}$-invariant partition into non-negligible subsets $X=\amalg X_{r}$, then

$$
\beta_{p}(\mathcal{R}, \mu)=\sum_{r} \mu\left(X_{r}\right) \beta_{p}\left(\mathcal{R} \mid X_{r}, \frac{\mu \mid X_{r}}{\mu\left(X_{r}\right)}\right) .
$$

Indeed, the Hilbert module $H$ one has to consider to define the $L^{2}$-Betti numbers may be decomposed into a direct sum $H=\bigoplus_{r} H_{r}$ according to the decomposition of $X$, and the normalization of the trace leads to $\operatorname{dim}_{\mathcal{R}} H_{r}=\mu\left(X_{r}\right) \operatorname{dim}_{\mathcal{R} \mid X_{r}} H_{r}$, and the formula. Thus, up to partitioning the space into the Borel subsets $X_{r}$ where the classes have constant cardinal $r$, one may compute $\beta_{0}(\mathcal{R})$ under the assumption that the classes all have cardinal $r$. If $r=\infty$, then $\beta_{0}(\mathcal{R})=0$ ([Gab02a], [Prop. 3.15). If $r$ is finite, there is a contractible $\mathcal{R}$-complex containing only one point in each fiber. The computation is then immediate, $\beta_{0}(\mathcal{R})=\frac{1}{r}$. In general,

$$
\beta_{0}(\mathcal{R}, \mu)=\sum_{r} \mu\left(X_{r}\right) \frac{1}{r}=\int_{X} \frac{d \mu(x)}{\# \mathcal{R}(x)} .
$$

Properties 2 and 3 follow. Property 4 is a consequence of formula 22 and [Gab02a], Th. 5.3.

As for Property 5, it is quite technical and we go back to the definitions in [Gab02a]. Observe that if the classes of $\mathcal{R}=\mathcal{R}_{1} * \mathcal{R}_{2}$ are almost all finite, then we are in a treeable situation and the formula is immediate by [Gab02a], Cor. 3.23, and [Gab00], Th. IV.15.

Recall that the first two $L^{2}$-Betti numbers $\left(\beta_{0}\right.$ and $\left.\beta_{1}\right)$ are computed by considering any 2 -dimensional simplicial $\mathcal{R}$-complex $\Sigma$ with simply connected fibers, and any exhausting increasing family of uniformly locally bounded $\mathcal{R}$-invariant subcomplexes $\left(\Sigma_{t}\right)_{t \in \mathbb{N}}$. Then, one calculate the limits of the von Neumann dimensions (with respect to the von Neumann algebra associated with $\mathcal{R}$ and the trace associated with $\mu$ ) for $*=0,1$ :

$$
\beta_{*}(\mathcal{R})=\lim _{s \rightarrow \infty} \lim _{t \rightarrow \infty, t \geq s} \underbrace{\operatorname{dim}_{\mathcal{R}} \operatorname{Clos}\left\{\operatorname{Im}\left(\bar{H}_{*}^{(2)}\left(\Sigma_{s}\right) \stackrel{J_{*, s, t}}{\rightarrow} \bar{H}_{*}^{(2)}\left(\Sigma_{t}\right)\right)\right\}}_{:=\nabla_{*}\left(\Sigma_{s}, \Sigma_{t}\right)}
$$

where $J_{*, s, t}$ is induced in homology by the inclusion $\Sigma_{s} \subset \Sigma_{t}$.

From

$\operatorname{Ker} \partial_{1}\left|C_{1}^{(2)}\left(\Sigma_{s}\right) \hookrightarrow \operatorname{Ker} \partial_{1}\right| C_{1}^{(2)}\left(\Sigma_{t}\right) \rightarrow \operatorname{Ker} \partial_{1} \mid C_{1}^{(2)}\left(\Sigma_{t}\right) / \operatorname{Clos}\left\{\partial_{2} C_{2}^{(2)}\left(\Sigma_{t}\right)\right\}$, 
and similarly in dimension 0 : with $\bar{H}_{0}^{(2)}\left(\Sigma_{s}\right)=C_{0}^{(2)}\left(\Sigma_{s}\right) / \operatorname{Clos}\left\{\partial_{1} C_{1}^{(2)}\left(\Sigma_{t}\right)\right\}$, we obtain that $\operatorname{Clos}\left\{\operatorname{Im} J_{1, s, t}\right\} \simeq \operatorname{Ker} \partial_{1} \mid C_{1}^{(2)}\left(\Sigma_{s}\right) / \operatorname{Clos}\left\{\partial_{2} C_{2}^{(2)}\left(\Sigma_{t}\right)\right\} \cap C_{1}^{(2)}\left(\Sigma_{s}\right)$ and $\operatorname{Clos}\left\{\operatorname{Im} J_{0, s, t}\right\}=C_{0}^{(2)}\left(\Sigma_{s}\right) / \operatorname{Clos}\left\{\partial_{1} C_{1}^{(2)}\left(\Sigma_{t}\right)\right\} \cap C_{0}^{(2)}\left(\Sigma_{s}\right)$. It follows that

$$
\begin{aligned}
& \nabla_{1}\left(\Sigma_{s}, \Sigma_{t}\right)=\operatorname{dim}_{\mathcal{R}} \operatorname{Ker} \partial_{1} \mid C_{1}^{(2)}\left(\Sigma_{s}\right)-\operatorname{dim}_{\mathcal{R}} \operatorname{Clos}\left\{\partial_{2} C_{2}^{(2)}\left(\Sigma_{t}\right)\right\} \cap C_{1}^{(2)}\left(\Sigma_{s}\right), \\
& \nabla_{0}\left(\Sigma_{s}, \Sigma_{t}\right)=\operatorname{dim}_{\mathcal{R}} C_{0}^{(2)}\left(\Sigma_{s}\right)-\operatorname{dim}_{\mathcal{R}} \operatorname{Clos}\left\{\partial_{1} C_{1}^{(2)}\left(\Sigma_{t}\right)\right\} \cap C_{0}^{(2)}\left(\Sigma_{s}\right) .
\end{aligned}
$$

Assuming $\Sigma_{s}=\mathcal{R}$ and $\beta_{0}\left(\Sigma_{s}\right)=\beta_{0}(\mathcal{R})$ for all $s$, (and we will check below that this can be assumed) the formula (25) becomes

$$
\begin{aligned}
\nabla_{0}\left(\Sigma_{s}, \Sigma_{t}\right) & =1-\operatorname{dim}_{\mathcal{R}} \operatorname{Clos}\left\{\partial_{1} C_{1}^{(2)}\left(\Sigma_{s}\right)\right\} \\
& =1-\left[\operatorname{dim}_{\mathcal{R}} C_{1}^{(2)}\left(\Sigma_{s}\right)-\operatorname{dim}_{\mathcal{R}} \operatorname{Ker} \partial_{1} \mid C_{1}^{(2)}\left(\Sigma_{s}\right)\right]
\end{aligned}
$$

and eventually

$$
\begin{aligned}
& \nabla_{1}\left(\Sigma_{s}, \Sigma_{t}\right)-\nabla_{0}\left(\Sigma_{s}, \Sigma_{t}\right) \\
& \quad=\operatorname{dim}_{\mathcal{R}} C_{1}^{(2)}\left(\Sigma_{s}\right)-1-\operatorname{dim}_{\mathcal{R}} \operatorname{Clos}\left\{\partial_{2} C_{2}^{(2)}\left(\Sigma_{t}\right)\right\} \cap C_{1}^{(2)}\left(\Sigma_{s}\right) .
\end{aligned}
$$

Recall that a simple graphing $\Phi$ made of partial isomorphisms taken from the full pseudogroup [[R]] naturally defines a 1-dimensional simplicial $\mathcal{R}$-complex $\Sigma_{\Phi}$ with 0 -skeleton $\Sigma_{\Phi}^{(0)}=\mathcal{R}$, where $\mathcal{R}$ is fibered by $\pi: \mathcal{R} \rightarrow X,(x, y) \mapsto x$ (see [Gab02a], Ex. 2.2.2). Conversely, if $\Sigma$ is a simplicial $\mathcal{R}$-complex with 0-skeleton $\Sigma^{(0)}=\mathcal{R}$, then its 1-skeleton $\Sigma^{(1)}$ defines the subrelation $\mathcal{R}_{\Sigma}$ of $\mathcal{R}$ where $(u, v) \in \mathcal{R}_{\Sigma}$ iff for some $x \in X,(x, u)$ and $(x, v)$ (seen as 0 -cells of $\Sigma$ ) belong to the same connected component of the $x$-fiber of $\Sigma$. In particular, $\mathcal{R}_{\Sigma}=\mathcal{R}$ iff $\Sigma$ is fiber-connected. Moreover if $\Sigma^{\prime} \subset \Sigma$ then $\mathcal{R}_{\Sigma^{\prime}} \subset \mathcal{R}_{\Sigma}$.

A (fiber)-simply connected 2-dimensional simplicial $\mathcal{R}$-complex $\Sigma$ with $\Sigma^{(0)}=$ $\mathcal{R}$ can be obtained from the fibering $\pi$ by taking $\Sigma^{(1)}$ to be the complete graph in each $\pi$-fiber and then adding a 2-cell for each triple of vertices in the $\pi$-fibers.

We claim that a fiber-connected simplicial $\mathcal{R}$-complex $\Sigma$ with $\Sigma^{(0)}=\mathcal{R}$ admits an exhausting increasing family of uniformly locally bounded $\mathcal{R}$-invariant sub-complexes $\left(\Sigma_{t}\right)_{t \in \mathbb{N}}$ (with $\Sigma_{t}^{(0)}=\mathcal{R}$ and) with $\beta_{0}\left(\Sigma_{t}, \mu\right)=\beta_{0}(\mathcal{R}, \mu)$. Since $\beta_{0}\left(\Sigma_{t}, \mu\right)=\beta_{0}\left(\mathcal{R}_{\Sigma_{t}}, \mu\right)$, in view of Proposition 6.1(1) it is enough to have $\# \mathcal{R}_{\Sigma_{t}}(x)=\# \mathcal{R}(x)$ for a.e. $x \in X$. And by monotonicity, this is satisfied for each $t$ as soon as it is satisfied for $t=0$. We restrict to the subset of $X$ made of the infinite $\mathcal{R}$-classes (since $\Sigma_{0}$ is obvious to construct on its complement). By [Zim84], 9.3.2, let $\mathcal{R}_{0}$ be an aperiodic hyperfinite subrelation, $\Phi_{0}$ a treeing of it with valency $\leq 3$ and $\Sigma_{0}:=\Sigma_{\Phi_{0}}$.

After this preparation, we resume considering an aperiodic free-product decomposed $\mathcal{R}=\mathcal{R}_{1} * \mathcal{R}_{2}$. We consider two 2-dimensional fiber-simply connected complexes and their exhaustions $\Sigma_{1},\left(\Sigma_{1, t}\right)_{t \in \mathbb{N}}$ for $\mathcal{R}_{1}$ and $\Sigma_{2},\left(\Sigma_{2, t}\right)_{t \in \mathbb{N}}$ for $\mathcal{R}_{2}$, such that $\Sigma_{1}^{(0)}=\Sigma_{1, t}^{(0)}=\mathcal{R}_{1}$ and $\Sigma_{2}^{(0)}=\Sigma_{2, t}^{(0)}=\mathcal{R}_{2}$. and such that 
$\beta_{0}\left(\Sigma_{1, t}, \mu\right)=\beta_{0}\left(\mathcal{R}_{1}, \mu\right)$ and $\beta_{0}\left(\Sigma_{2, t}, \mu\right)=\beta_{0}\left(\mathcal{R}_{2}, \mu\right)$. By suspension [Gab02a], Sect. 5.2, we get the induced $\mathcal{R}$-complexes $\widetilde{\Sigma}_{1},\left(\widetilde{\Sigma}_{1, t}\right)_{t \in \mathbb{N}}$ and $\widetilde{\Sigma}_{2},\left(\widetilde{\Sigma}_{2, t}\right)_{t \in \mathbb{N}}$ (or equivalently since $\Sigma_{i}^{(0)}=\mathcal{R}_{i} \subset \mathcal{R}$, we consider the $\mathcal{R}$-saturations of the various $\mathcal{R}_{i}$-complexes).

The 2-dimensional simplicial $\mathcal{R}$-complex $\widetilde{\Sigma}=\widetilde{\Sigma}_{1} \cup \widetilde{\Sigma}_{2}$ has 0-skeleton $=\mathcal{R}$ and exhausting sequence $\widetilde{\Sigma}_{t}=\widetilde{\Sigma}_{1, t} \cup \widetilde{\Sigma}_{2, t}$. It is fiber-connected: its 1-skeleton generates the equivalence relation $\mathcal{R}_{\widetilde{\Sigma}}=\mathcal{R}_{\widetilde{\Sigma}_{1}} \vee \mathcal{R}_{\widetilde{\Sigma}_{2}}=\mathcal{R}_{1} \vee \mathcal{R}_{2}=\mathcal{R}$; and it is fiber-simply connected: a simple loop (pushed by homotopy) in the 1-skeleton of a fiber gives a $n$-tuple of points in $X$ that are $\mathcal{R}_{\widetilde{\Sigma}_{1}}$ - or $\mathcal{R}_{\widetilde{\Sigma}_{2}}$-equivalent and the free product hypothesis implies that the loop is entirely contained in one (simply connected) connected component of one of the $\widetilde{\Sigma}_{j}$.

We claim that $\beta_{0}\left(\widetilde{\Sigma}_{t}\right)=\beta_{0}\left(\mathcal{R}_{1} * \mathcal{R}_{2}\right)$ for all $t \in \mathbb{N}$ : a finite $\mathcal{R}_{\widetilde{\Sigma}_{t}}$-class decomposes into its (finite) $\mathcal{R}_{\widetilde{\Sigma}_{1, t}}$ and $\mathcal{R}_{\widetilde{\Sigma}_{2, t}}$-classes that are complete, i.e. $\mathcal{R}_{\widetilde{\Sigma}_{j, t}}(y)=$ $\mathcal{R}_{j}(y)$, by the assumption $\beta_{0}\left(\widetilde{\Sigma}_{j, t}\right)=\beta_{0}\left(\mathcal{R}_{j}\right)$.

Thanks to the particular shape of $\widetilde{\Sigma}$, the boundary operator $\partial_{2}$ decomposes by blocks:

$$
C_{2}^{(2)}(\tilde{\Sigma})=C_{2}^{(2)}\left(\widetilde{\Sigma}_{1}\right) \oplus C_{1}^{(2)}\left(\widetilde{\Sigma}_{2}\right) \stackrel{\left(\partial_{2}\right)_{1} \oplus\left(\partial_{2}\right)_{2}}{\rightarrow} C_{1}^{(2)}\left(\widetilde{\Sigma}_{1}\right) \oplus C_{1}^{(2)}\left(\widetilde{\Sigma}_{2}\right)=C_{1}^{(2)}(\tilde{\Sigma}) .
$$

The terms in the right hand side of the formula (26) for $\tilde{\Sigma}$ may be split accordingly.

It follows that

$$
\begin{aligned}
\nabla_{1}\left(\widetilde{\Sigma}_{s}, \widetilde{\Sigma}_{t}\right)-\nabla_{0}\left(\widetilde{\Sigma}_{s}, \widetilde{\Sigma}_{t}\right)= & \nabla_{1}\left(\widetilde{\Sigma}_{1, s}, \widetilde{\Sigma}_{1, t}\right)-\nabla_{0}\left(\widetilde{\Sigma}_{1, s}, \widetilde{\Sigma}_{1, t}\right)-1 \\
& +\left(\nabla_{1}\left(\widetilde{\Sigma}_{2, s}, \widetilde{\Sigma}_{2, t}\right)-\nabla_{0}\left(\widetilde{\Sigma}_{2, s}, \widetilde{\Sigma}_{2, t}\right)-1\right)+1
\end{aligned}
$$

and taking the limits, like in (24), leads to the required formula of Property 5.

6.2. Proof of Theorem 4.19. Consider $\mathcal{R}$ with $\beta_{1}(\mathcal{R})=0$ and all the classes infinite. We start assuming that $\mathcal{R}$ decomposes as a free product of two factors $\mathcal{R}=\mathcal{R}_{1} * \mathcal{R}_{2}$. Let $U_{i}$ be the union of the infinite $\mathcal{R}_{i}$-classes, for $i=1,2$.

1. We show that $\mathcal{R}\left|U_{1}=\mathcal{R}_{1}\right| U_{1}, \mathcal{R}\left|U_{2}=\mathcal{R}_{2}\right| U_{2}$ and both $\mathcal{R}_{2} \mid U_{1}$ and $\mathcal{R}_{1} \mid U_{2}$ are trivial: If $\mu\left(U_{1}\right)>0$, then the restrictions of $\mathcal{R}$ and $\mathcal{R}_{1}$ to $U_{1}$ satisfy respectively $\beta_{1}\left(\mathcal{R} \mid U_{1}\right)=0$ (by 6.1 Property 4) and $\beta_{0}\left(\mathcal{R}_{1} \mid U_{1}\right)=0$ (by 6.1 Property 3). By Theorem 3.2, $\mathcal{R}\left|U_{1}=\mathcal{R}_{1}\right| U_{1} * S$, where we have isolated the particular subrelation $\mathcal{V}_{i_{1}}=\mathcal{R}_{1} \cap \mathcal{R}\left|U_{1}=\mathcal{R}_{1}\right| U_{1}$ (Theorem 3.2(2)) and we have put all the other terms of the free product decomposition together to form $\delta$, which itself contains the other subrelation $\mathcal{V}_{i_{2}}=\mathcal{R}_{2} \cap \mathcal{R}\left|U_{1}=\mathcal{R}_{2}\right| U_{1}$.

By 6.1 Property $5, \underbrace{\beta_{1}\left(\mathcal{R} \mid U_{1}\right)}_{=0}=\underbrace{\beta_{1}\left(\mathcal{R}_{1} \mid U_{1}\right)}_{\geq 0}+\underbrace{\beta_{1}(\mathcal{S})}_{\geq 0}+1-(\underbrace{\beta_{0}\left(\mathcal{R}_{1} \mid U_{1}\right)}_{=0}+\beta_{0}(\mathcal{S}))$ so that $\beta_{0}(\mathcal{S})=1$, i.e. (by Proposition 6.1, item 2) $S$ is trivial. It follows that $\mathcal{R}_{2} \mid U_{1}$ is trivial and the decomposition reduces to $\mathcal{R}\left|U_{1}=\mathcal{R}_{1}\right| U_{1}$. Symmetrically, if $\mu\left(U_{2}\right)>0, \mathcal{R}_{1} \mid U_{2}$ is trivial and $\mathcal{R}\left|U_{2}=\mathcal{R}_{2}\right| U_{2}$. 
2. We claim that $\mu\left(U_{1} \cap U_{2}\right)=0$, for otherwise, $\left(\mathcal{R}_{1} \mid U_{1}\right) \mid U_{1} \cap U_{2}$ the iterated restriction would have infinite classes (by the following standard lemma). But it is also the trivial subrelation $\left(\mathcal{R}_{1} \mid U_{2}\right) \mid U_{1} \cap U_{2}$.

Lemma 6.2. If the p.m.p. equivalence relation $\mathcal{R}$ is aperiodic, and $V \subset X$ is nonnegligible, then the restriction $\mathcal{R} \mid V$ is aperiodic.

3. We claim that the partition $U=U_{1} \amalg U_{2}$ is $\mathcal{R} \mid U$-invariant. We may assume both are non-null. The partition is already $\mathcal{R}_{1} \mid U$ - and $\mathcal{R}_{2} \mid U$-invariant. As above, Theorem 3.2 gives a decomposition $\mathcal{R}\left|U=\mathcal{R}_{1}\right| U * S$, where $\delta$ contains $\mathcal{R}_{2} \mid U$. The above parts (1) and (2) apply to this decomposition, with $U_{2}^{\prime}$ the union of the infinite $\mathcal{S}$-classes, in place of $U_{2}$, leading to $\mu\left(U_{1} \cap U_{2}^{\prime}\right)=0$. Observe that $U_{2}^{\prime}$ contains the $\delta$-saturation of $U_{2}=U \backslash U_{1}$, so that $U_{2}^{\prime}=U_{2}$ (a.s.) and $U_{2}$ is $\delta$-invariant. Being also $\mathcal{R}_{1} \mid U$-invariant, $U_{2}$ ends up $\mathcal{R} \mid U$-invariant. Symmetrically, $U_{1}$ is $\mathcal{R} \mid U$-invariant.

The four first points of Theorem 4.19 have been proved for two factors.

4. If now $\mathcal{R}=\mathcal{R}_{1} * \mathcal{R}_{2} * \cdots * \mathcal{R}_{i} * \cdots$, we apply the above result after one factor $\mathcal{R}_{i}$ has been isolated and the other ones have been glued together in an $\overline{\mathcal{R}}_{i}$ leading to a decomposition $\mathcal{R}=\mathcal{R}_{i} * \overline{\mathcal{R}}_{i}$. The union $\bar{U}_{i}$ of the infinite orbits of $\overline{\mathcal{R}}_{i}$ contains all the $U_{j}$, for $j \neq i$. We immediately deduce the four first points of Theorem 4.19 in general. For instance, $U_{i}$ being $\mathcal{R} \mid U_{i} \cup \bar{U}_{i}$-invariant is also invariant for the even more restricted equivalence relation $\mathcal{R} \mid\left(U_{1} \cup U_{2} \cup \cdots \cup U_{i} \cup \cdots\right)$.

5. We conclude by proving that the restriction of $\mathcal{R}$ to $W=X \backslash \mathcal{R} . U$, the complement of the saturation of $U$, is hyperfinite as soon as $\mu(W)>0$. Observe that $W$ is $\mathcal{R}$-invariant and that $\mathcal{R}\left|W=\mathcal{R}_{1}\right| W * \mathcal{R}_{2}\left|W * \cdots * \mathcal{R}_{i}\right| W * \cdots$. By definition of $U$, the restrictions $\mathcal{R}_{i} \mid W$ are finite subrelations, and thus treeable, so that $\mathcal{R} \mid W$ follows treeable and aperiodic. Since moreover $\beta_{1}(\mathcal{R} \mid W)=0$ (Proposition 6.1 Property 4 ) it is hyperfinite by Proposition 6.10 of [Gab02a]. If $\mathcal{R}$ is nowhere hyperfinite, then $\mu(W)=0$ and $U$ is a complete section for $\mathcal{R}$. This completes the proof of Theorem 4.19.

6.3. Proof of Theorem 5.1. By Theorem 3.1 and Proposition 4.10, the proof reduces to the case where $\Theta: \mathrm{D}(\mathcal{R}) \rightarrow \mathrm{D}\left(\mathcal{R}^{\prime}\right)$ is in fact an OE between $\mathcal{R}=\boldsymbol{*}_{p \in P} \mathcal{R}_{p} * \mathcal{T}$ and $\mathcal{R}^{\prime}=*_{p^{\prime} \in P^{\prime}} \mathcal{R}_{p^{\prime}}^{\prime} * \mathcal{T}^{\prime}$.

a) Fix one $p \in P$ and define the subrelation

$$
\mathcal{E}_{p}^{\prime}:=\Theta \mathcal{R}_{p} \Theta^{-1}
$$

of $\mathcal{R}^{\prime}$, the image of $\mathcal{R}_{p}$ under $\Theta$. It admits a decomposition according to Theorem 3.2:

$$
\left.\mathcal{E}_{p}^{\prime}=\underset{p^{\prime} \in P^{\prime}}{*} \underset{k^{\prime} \in K^{\prime}\left(p, p^{\prime}\right)}{*} \underset{\mathcal{V}^{\prime}}{*}\right) * \mathcal{T}_{p}^{\prime}
$$

where for each $k^{\prime} \in K^{\prime}\left(p, p^{\prime}\right)$,

$$
\mathcal{V}_{k^{\prime}}^{\prime}=\mathcal{E}_{p}^{\prime} \cap \psi_{k^{\prime}}^{\prime-1} \mathcal{R}_{p^{\prime}}^{\prime} \psi_{k^{\prime}}^{\prime}
$$


with $\psi_{k}^{\prime} \in\left[\left[\mathcal{R}^{\prime}\right]\right]$ a partial isomorphism defined on $\mathrm{D}\left(\mathcal{V}_{k^{\prime}}^{\prime}\right)$. In particular, $\mathcal{V}_{k^{\prime}}^{\prime}$ is inner conjugate in $\mathcal{R}^{\prime}$ with a subrelation of $\mathcal{R}_{p^{\prime}}^{\prime}$. As for $\mathcal{T}_{p}^{\prime}$, it is a treeable subrelation, containing the treeable part given by Theorem 3.2 and the conjugates of subrelations of $\mathcal{T}^{\prime}$ (see Remark 3.4).

Since $\mathcal{E}_{p}^{\prime}$ is $\mathcal{F} \mathcal{I}$ just like $\mathcal{R}_{p}$, this decomposition (28) admits a trivializing partition (Definition 4.1). Observe that the treeable part $\mathcal{T}_{p}^{\prime}$ cannot survive as a slice in the trivializing partition: its restriction to some $U_{i}$ would coincide with $\varepsilon_{p}^{\prime} \mid U_{i}$, would be treeable [Gab00], Prop. II.6, and (F्FI) (Proposition 4.10); and thus smooth (Proposition 4.6), which is ruled out by the aperiodicity assumption on $\mathcal{R}_{p}$. The trivializing partition thus takes the form

$$
\coprod_{p^{\prime} \in P^{\prime}} \coprod_{k^{\prime} \in K^{\prime}\left(p, p^{\prime}\right)} U_{k^{\prime}}^{\prime}
$$

and induces the slicing (see Remark 4.3 item 2, equation (21)) affiliated with the partition of $\mathrm{D}\left(\mathcal{E}_{p}^{\prime}\right)$ into the $\mathcal{E}_{p}^{\prime}$-saturations $V_{k^{\prime}}^{\prime}$ of $U_{k^{\prime}}^{\prime}$

$$
\begin{aligned}
\mathcal{E}_{p}^{\prime} & =\underset{p^{\prime} \in P^{\prime}}{*} \underset{k^{\prime} \in K^{\prime}\left(p, p^{\prime}\right)}{*} \underset{\mathcal{E}_{p}^{\prime}}{*} V_{k^{\prime}}^{\prime} \\
\mathcal{R}_{p}=\Theta^{-1} \mathcal{E}_{p}^{\prime} \Theta & =\underset{p^{\prime} \in P^{\prime}}{*} \underset{k^{\prime} \in K^{\prime}\left(p, p^{\prime}\right)}{*} \mathcal{R}_{p} \mid \Theta^{-1} V_{k^{\prime}}^{\prime}
\end{aligned}
$$

Moreover, by definition of the trivialization, for each $k^{\prime} \in K^{\prime}\left(p, p^{\prime}\right)$ the restriction of $\mathcal{E}_{p}^{\prime}$ to $U_{k^{\prime}}^{\prime}$ satisfies:

$$
\begin{aligned}
\mathcal{E}_{p}^{\prime} \mid U_{k^{\prime}}^{\prime} & =\mathcal{V}_{k^{\prime}}^{\prime} \mid U_{k^{\prime}}^{\prime} \\
& =\left(\mathcal{E}_{p}^{\prime} \cap\left(\psi^{\prime} k^{\prime} \mathcal{R}_{p^{\prime}}^{\prime} \psi_{k^{\prime}}^{\prime}\right)\right) \mid U_{k^{\prime}}^{\prime} \\
& =\mathcal{E}_{p}^{\prime}\left|U_{k^{\prime}}^{\prime} \cap\left(\psi^{\prime}{ }_{k^{\prime}}^{-1} \mathcal{R}_{p^{\prime}}^{\prime} \psi_{k^{\prime}}^{\prime}\right)\right| U_{k^{\prime}}^{\prime}
\end{aligned}
$$

which means exactly

$$
\mathcal{E}_{p}^{\prime}\left|U_{k^{\prime}}^{\prime} \subset\left(\psi_{k^{\prime}}^{\prime-1} \mathcal{R}_{p^{\prime}}^{\prime} \psi_{k^{\prime}}^{\prime}\right)\right| U_{k^{\prime}}^{\prime}
$$

Since we did not yet use the properties of the $\mathcal{R}_{p^{\prime}}^{\prime}$, let us raise what we proved so far:

Proposition 6.3. If $\mathcal{S}=\boldsymbol{*}_{q \in Q} \mathcal{S}_{q} * \mathcal{T}$ is p.m.p. and $\mathcal{T}$ is treeable, and if $\mathcal{E}$ is a subrelation that is freely indecomposable and aperiodic on its domain, then there are (at most) countably many disjoint Borel subsets $U_{r}$ whose $\mathcal{E}$-saturations $V_{r}=\mathcal{E} U_{r}$ form an $\mathcal{E}$-invariant partition $\mathrm{D}(\mathcal{E})=\bigsqcup_{q \in Q} \bigsqcup_{r \in R(q)} V_{r}$ with affiliated slicing $\mathcal{E}=$ $*_{q \in Q} *_{r \in R(q)} \mathcal{E} \mid V_{r}$ and such that for $r \in R(q)$, one has $\mathcal{E}\left|U_{r} \subset \psi_{r}^{-1} \oint_{q} \psi_{r}\right| U_{r}$ for some $\psi_{r} \in[[S]]$. 
b) Observe that the slicings (31) of the factors $\mathcal{E}_{p}^{\prime}:=\Theta \mathcal{R}_{p} \Theta^{-1}$ induce the corresponding free product decompositions of $\mathcal{R}^{\prime}=\Theta \mathcal{R} \Theta^{-1}$ and $\mathcal{R}$ :

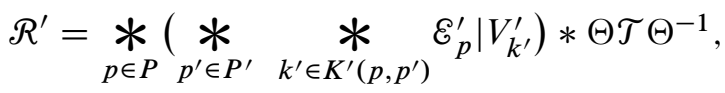

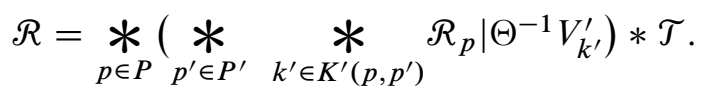

c) The subrelation $\left(\psi_{k^{\prime}}^{\prime-1} \mathcal{R}_{p^{\prime}}^{\prime} \psi_{k^{\prime}}^{\prime}\right) \mid U_{k^{\prime}}^{\prime}$ of $\mathcal{R}^{\prime}=\Theta \mathcal{R} \Theta^{-1}$ appearing in (33), for some $p \in P, p^{\prime} \in P^{\prime}$ and $k^{\prime} \in K^{\prime}\left(p, p^{\prime}\right)$ such that $U_{k^{\prime}}^{\prime}$ is non-negligible, gets itself a free product decomposition with respect to (34), given by Theorem 3.2:

$$
\left(\psi_{k^{\prime}}^{\prime-1} \mathcal{R}_{p^{\prime}}^{\prime} \psi_{k^{\prime}}^{\prime}\right) \mid U_{k^{\prime}}^{\prime}=\left(* l \in L \mathcal{W}_{l}\right) * \mathcal{T}_{k^{\prime}} .
$$

The point 2 of Theorem 3.2 states that the particular term

$$
\mathcal{W}_{l_{0}}=\left(\psi^{\prime-1} \mathcal{R}_{p^{\prime}}^{\prime} \psi_{k^{\prime}}^{\prime}\right)\left|U_{k^{\prime}}^{\prime} \cap \mathcal{E}_{p}^{\prime}\right| V_{k^{\prime}}^{\prime}
$$

has to appear and from (33), we get

$$
W_{l_{0}}=\varepsilon_{p}^{\prime} \mid U_{k^{\prime}}^{\prime}
$$

On the other hand, $\left(\psi^{\prime}{ }_{k^{\prime}}^{-1} \mathcal{R}_{p^{\prime}}^{\prime} \psi_{k^{\prime}}^{\prime}\right) \mid U_{k^{\prime}}^{\prime}$ is $\mathcal{F} \mathcal{I}$ since isomorphic with the restriction of the $\mathcal{F} \mathcal{I}$ relation $\mathcal{R}_{p^{\prime}}^{\prime}$ to a non-null subset of its domain. As such, its decomposition (36) admits a trivialization.

But the particular term $\varepsilon_{p}^{\prime} \mid U_{k^{\prime}}^{\prime}$ is nowhere smooth on its whole domain $U_{k^{\prime}}^{\prime}$, so that (Proposition 4.4 item 3 ) this term is the only one of the decomposition (36); i.e. (33) is an equality:

$$
\begin{aligned}
\mathcal{E}_{p}^{\prime} \mid U_{k^{\prime}}^{\prime} & =\left(\psi_{k^{\prime}}^{\prime-1} \mathcal{R}_{p^{\prime}}^{\prime} \psi_{k^{\prime}}^{\prime}\right) \mid U_{k^{\prime}}^{\prime}, \\
\Theta \mathcal{R}_{p}\left|\Theta^{-1}\left(U_{k^{\prime}}^{\prime}\right) \Theta^{-1}=\left(\Theta \mathcal{R}_{p} \Theta^{-1}\right)\right| U_{k^{\prime}}^{\prime} & =\psi_{k^{\prime}}^{\prime-1} \mathcal{R}_{p^{\prime}}^{\prime} \mid \psi_{k^{\prime}}^{\prime}\left(U_{k^{\prime}}^{\prime}\right) \psi_{k^{\prime}}^{\prime}
\end{aligned}
$$

This shows that the map $\psi_{k^{\prime}}^{\prime} \Theta$ defines, for $k^{\prime} \in K^{\prime}\left(p, p^{\prime}\right)$, an isomorphism

$$
\psi_{k^{\prime}}^{\prime} \Theta: \mathcal{R}_{p}\left|\Theta^{-1}\left(U_{k^{\prime}}^{\prime}\right) \stackrel{\mathrm{OE}}{\sim} \mathcal{R}_{p^{\prime}}^{\prime}\right| \psi_{k^{\prime}}^{\prime}\left(U_{k^{\prime}}^{\prime}\right)
$$

and thus a SOE between the slicing term $\mathcal{R}_{p}\left|\Theta^{-1}\left(V_{k^{\prime}}^{\prime}\right)=\Theta^{-1} \mathcal{E}_{p}^{\prime}\right| V_{k^{\prime}}^{\prime} \Theta$ of (32) and $\mathcal{R}_{p^{\prime}}^{\prime} \mid \psi_{k^{\prime}}^{\prime}\left(U_{k^{\prime}}^{\prime}\right)$, the restriction of $\mathcal{R}_{p^{\prime}}^{\prime}$ to $\psi_{k^{\prime}}^{\prime}\left(U_{k^{\prime}}^{\prime}\right)$,

$$
\psi_{k^{\prime}}^{\prime} \Theta: \mathcal{R}_{p}\left|\Theta^{-1}\left(V_{k^{\prime}}^{\prime}\right) \stackrel{\operatorname{SOE}}{\sim} \mathcal{R}_{p^{\prime}}^{\prime}\right| \psi_{k^{\prime}}^{\prime}\left(U_{k^{\prime}}^{\prime}\right)
$$

d) Fix a $p^{\prime} \in P^{\prime}$. We will show that the family of sets

$$
W^{\prime}\left(k^{\prime}\right):=\psi_{k^{\prime}}^{\prime}\left(U_{k^{\prime}}^{\prime}\right) \quad \text { for } k^{\prime} \in \coprod_{p \in P} K^{\prime}\left(p, p^{\prime}\right)
$$


induces a slicing of $\mathcal{R}_{p^{\prime}}^{\prime}$ (in particular the family is not empty), i.e. we show that their $\mathcal{R}_{p^{\prime}}^{\prime}$-saturation form a partition of $\mathrm{D}\left(\mathcal{R}_{p^{\prime}}^{\prime}\right)$.

d-1) We first show that their $\mathcal{R}_{p^{\prime}}^{\prime}$-saturation intersect trivially. Let $k_{1}^{\prime} \in K^{\prime}\left(p_{1}, p^{\prime}\right)$ and $k_{2}^{\prime} \in K^{\prime}\left(p_{2}, p^{\prime}\right)$ such that the $\mathcal{R}_{p^{\prime}}^{\prime}$-saturations of $W^{\prime}\left(k_{1}^{\prime}\right)$ and $W^{\prime}\left(k_{2}^{\prime}\right)$ have a non-null intersection, i.e. there is a partial isomorphism $\rho^{\prime} \in\left[\left[\mathcal{R}_{p^{\prime}}^{\prime}\right]\right]$ with (nonnull) domain contained in $W^{\prime}\left(k_{1}^{\prime}\right)$ and target in $W^{\prime}\left(k_{2}^{\prime}\right)$. It follows that the partial isomorphism $\psi_{k_{2}^{\prime}}^{\prime-1} \rho^{\prime} \psi_{k_{1}^{\prime}}^{\prime}$ has non-null domain $A_{1} \subset U_{k_{1}^{\prime}}^{\prime}$ and target $A_{2} \subset U_{k_{2}^{\prime}}^{\prime}$ and conjugates $\mathcal{E}_{p_{1}}^{\prime} \mid A_{1}$ with $\mathcal{E}_{p_{2}}^{\prime} \mid A_{2}$. But these subrelations are not smooth and appear as subrelations of factors of the free product decomposition (34). It follows from Lemma 2.3 that they cannot belong to different factors, i.e. $k_{1}^{\prime}=k_{2}^{\prime}$.

$\mathrm{d}$-2) Consider now the invariant partition of $\mathrm{D}\left(\mathcal{R}_{p^{\prime}}^{\prime}\right)$ given by the $\mathcal{R}_{p^{\prime}}^{\prime}$-saturations of the sets $W^{\prime}\left(k^{\prime}\right)$, for $k^{\prime} \in \bigsqcup_{p \in P} K^{\prime}\left(p, p^{\prime}\right)$, and the complement $Z\left(p^{\prime}\right)$ of their union in $\mathrm{D}\left(\mathcal{R}_{p^{\prime}}^{\prime}\right)$; and consider the affiliated slicing of $\mathcal{R}_{p^{\prime}}^{\prime}$. We will show that the measure of $Z\left(p^{\prime}\right)$ is zero. We exchange the roles of $\mathcal{R}$ and $\mathcal{R}^{\prime}$ after having further decomposed $\mathcal{R}^{\prime}$ thanks to the just above constructed slicing of $\mathcal{R}_{p^{\prime}}^{\prime}$ affiliated with $\bigsqcup_{p \in P} \bigsqcup_{k^{\prime} \in K^{\prime}\left(p, p^{\prime}\right)} \mathcal{R}_{p^{\prime}}^{\prime} W^{\prime}\left(k^{\prime}\right) \amalg Z\left(p^{\prime}\right)$ :

$$
\left.\mathcal{R}^{\prime}=\underset{p^{\prime} \in P^{\prime}}{*}\left(\underset{p \in P}{*} \underset{k^{\prime} \in K^{\prime}\left(p, p^{\prime}\right)}{*} \underset{\mathcal{R}^{\prime}}{*} \mid \mathcal{R}_{p^{\prime}}^{\prime} W^{\prime}\left(k^{\prime}\right)\right) * \mathcal{R}_{p^{\prime}}^{\prime} \mid Z\left(p^{\prime}\right)\right) * \mathcal{T}^{\prime} .
$$

We use $\Theta^{-1}$ and apply the above steps a), b), c). After a restriction of its domain, the slice $\mathcal{R}_{p^{\prime}}^{\prime} \mid Z\left(p^{\prime}\right)$ is conjugate with one of the $\mathcal{R}_{p}$ restricted to a Borel subset $Y$ of its domain, like in (37) via some $\eta_{1} \Theta^{-1}$, where $\eta_{1} \in[[\mathcal{R}]]$. This restriction $\mathcal{R}_{p} \mid Y$ has just been shown ((37) again but in the direct sense) to be conjugate (up to an additional restriction) with a restriction of one of the $\mathcal{R}_{q^{\prime}}^{\prime} \mid W^{\prime}\left(k^{\prime}\right)$, for some $q^{\prime} \in P^{\prime}$ and $k^{\prime} \in K^{\prime}\left(p, q^{\prime}\right)$, via some $\eta_{2}^{\prime} \Theta$, with $\eta_{2}^{\prime} \in\left[\left[\mathcal{R}^{\prime}\right]\right]$. Since the composition $\eta_{2}^{\prime} \Theta \eta_{1} \Theta^{-1} \in\left[\left[\mathcal{R}^{\prime}\right]\right]$, it follows that up to restricting to a non-negligible Borel subset, $\mathcal{R}_{p^{\prime}}^{\prime} \mid Z\left(p^{\prime}\right)$ is inner conjugate with a restriction of $\mathcal{R}_{q^{\prime}}^{\prime} \mid W^{\prime}\left(q^{\prime}\right)$, one of the factors in the decomposition of $\mathcal{R}^{\prime}$, which is different from $\mathcal{R}_{p^{\prime}}^{\prime} \mid Z\left(p^{\prime}\right)$ by definition of $Z\left(p^{\prime}\right)$. Lemma 2.3 would imply that $\mathcal{R}_{p^{\prime}}^{\prime} \mid Z\left(p^{\prime}\right)$ is somewhere smooth, contrarily to the assumption that the orbits of the $\mathcal{R}_{p^{\prime}}^{\prime}$ are all infinite on its domain. It follows that the measure of $Z\left(p^{\prime}\right)$ is zero.

The families $W^{\prime}\left(k^{\prime}\right)$ induce slicings of the factors $\mathcal{R}_{p^{\prime}}^{\prime}$ leading to a refined free product decomposition of $\mathcal{R}^{\prime}$ whose (non treeable) terms are indexed by $K:=$ $\bigsqcup_{p \in P} \bigsqcup_{p^{\prime} \in P^{\prime}} K^{\prime}\left(p, p^{\prime}\right)$ and in a bijective SOE correspondence with those of the refined decomposition (35) of $\mathcal{R}$, via $\Theta$ and inner partial isomorphisms. The slicing of $\mathcal{R}_{p}$ we were after in Theorem 5.1 is affiliated with the $A_{k^{\prime}}:=\Theta^{-1} V_{k^{\prime}}^{\prime}$ (the $\mathcal{R}_{p^{-}}$ saturation of the $\left.\Theta^{-1} U_{k^{\prime}}^{\prime}\right)$, for $k^{\prime} \in K(p):=\bigsqcup_{p^{\prime} \in P^{\prime}} K^{\prime}\left(p, p^{\prime}\right)$, while the slicing of $\mathcal{R}_{p^{\prime}}^{\prime}$ is affiliated with the $\mathcal{R}_{p^{\prime}}^{\prime}$-saturation $B_{k^{\prime}}:=\mathcal{R}_{k^{\prime}}^{\prime} W^{\prime}\left(k^{\prime}\right)=\mathcal{R}_{k^{\prime}}^{\prime} \psi_{k^{\prime}}^{\prime}\left(U_{k^{\prime}}^{\prime}\right)$, for $k^{\prime} \in K^{\prime}\left(p^{\prime}\right):=\bigsqcup_{p \in P} K^{\prime}\left(p, p^{\prime}\right)$, and the SOE is given by (37). 


\subsection{Proof of the corollaries}

Proof of Theorem 1.5 of the introduction. By measure equivalence with free groups, we have treeable free p.m.p. actions $\Gamma_{0} \curvearrowright^{\alpha} X$ and $\Lambda_{0} \curvearrowright^{\beta} Y$. Considering a coupling $(\Omega, v)$ witnessing the measure equivalence of Equation (6), the corresponding diagonal-action coupling on $\Omega \times X \times Y$, obtained by extending $\alpha, \beta$ trivially on the other factors, delivers SOE actions of $*_{i \in I} \Gamma_{i} * \Gamma_{0}$ and $*_{j \in J} \Lambda_{j} * \Lambda_{0}$ whose restrictions to $\Gamma_{0}$ and $\Lambda_{0}$ are treeable. Theorem 1.5 then follows immediately from Theorem 5.1.

Proof of Corollary 1.9. The ergodicity assumption on the $\alpha$ side prevents from any slicing for $\mathcal{R}_{\alpha}$ in Theorem 5.1 which gives nevertheless a bijection

$$
\theta: P \rightarrow \coprod_{p^{\prime} \in P^{\prime}} K^{\prime}\left(p^{\prime}\right)
$$

( $n=n^{\prime}$ follows) for which $\Theta$ induces a SOE of the shape $f^{\prime} \Theta f$, where $f^{\prime} \in\left[\left[\mathcal{R}_{\alpha^{\prime}}\right]\right]$ and $f \in\left[\left[\mathcal{R}_{\alpha}\right]\right]$, between the terms $\mathcal{R}_{\alpha \mid \Gamma_{p}}$ and $\mathcal{R}_{\alpha^{\prime} \mid \Gamma_{\theta(p)}^{\prime}}$. The latter follows ergodic. Under the moreover assumption, the free products $\boldsymbol{*}_{p \in P} \Gamma_{p}$ and $\boldsymbol{*}_{p^{\prime} \in P^{\prime}} \Gamma_{p^{\prime}}^{\prime}$ have the same first $\ell^{2}$-Betti number, $\neq 0, \infty$. Thus any SOE between them has to be an OE ([Gab02a]): $\Theta$ induces an OE between the ergodic subrelations $\mathcal{R}_{\alpha \mid \Gamma_{p}}$ and $\mathcal{R}_{\alpha^{\prime} \mid \Gamma_{\theta(p)}^{\prime}}$.

Corollary 1.11 of the introduction is a specialization of the following.

Theorem 6.4. Let $\Theta$ be a SOE between two actions $\alpha$ and $\alpha^{\prime}$ as in Framework 1.7. Assume that $\Gamma_{1} \stackrel{\mathrm{ME}}{\sim} \Gamma_{1}^{\prime}$ with generalized index 1 , and that $\Gamma_{1} \stackrel{\mathrm{ME}}{\chi} \Gamma_{p}, \Gamma_{p^{\prime}}^{\prime}$ for all $p, p^{\prime} \neq 1$. Assume moreover that $I_{\mathrm{ME}}\left(\Gamma_{1}\right)=\{1\}$. Then $\Theta$ is in fact an orbit equivalence and the restrictions to $\Gamma_{1}$ and $\Gamma_{1}^{\prime}$ are $O E$. In particular, they have the same measure space of ergodic components, in particular the same families of measures of ergodic components (possibly with repetition).

Proof. Theorem 5.1 applied to the SOE $\Theta$ between $\mathcal{R}_{\alpha}=\mathcal{*}_{p \in P} \mathcal{R}_{\alpha \mid \Gamma_{p}} * \mathcal{R}_{\alpha \mid \Gamma_{0}}$ and $\mathcal{R}_{\alpha^{\prime}}=*_{p^{\prime} \in P^{\prime}} \mathcal{R}_{\alpha}^{\prime} \mid \Gamma_{p^{\prime}}^{\prime} * \mathcal{R}_{\alpha^{\prime} \mid \Gamma_{0}^{\prime}}$ produces slicings of $\mathcal{R}_{\alpha \mid \Gamma_{1}}$ and $\mathcal{R}_{\alpha^{\prime} \mid \Gamma_{1}^{\prime}}$ whose components are pairwise associated by $\theta$ and SOE via partial isomorphisms of the shape $f^{\prime} \Theta f$ with $f^{\prime}, f$ preserving respectively the measures $\mu, \mu^{\prime}$. They all scale the measure by the same factor and may be assembled together in order to produce a global SOE between $\mathcal{R}_{\alpha \mid \Gamma_{1}}$ and $\mathcal{R}_{\alpha^{\prime} \mid \Gamma_{1}^{\prime}}$, with the same compression constant. The point being that all together the slices meet almost all their classes. Now $I_{\mathrm{ME}}\left(\Gamma_{1}\right)=\{1\}$ (for instance if some $\left.\beta_{p}\left(\Gamma_{1}\right) \neq 0, \infty\right)$ and $\Gamma_{1} \underset{1}{\stackrel{\mathrm{ME}}{\sim}} \Gamma_{1}^{\prime}$ imply that any SOE between free p.m.p. $\Gamma_{1}$ - and $\Gamma_{1}^{\prime}$-actions is in fact an OE. The compression constant equals 1 and $\Theta$ is in fact an OE. 


\section{References}

[Ada88] S. Adams, Indecomposability of treed equivalence relations. Israel J. Math. 64 (1988), 362-380 (1989). Zbl 0678.28010 MR 995576

[Ada90] S. Adams, Trees and amenable equivalence relations. Ergodic Theory Dynam. Systems 10 (1990), 1-14. Zbl 0667.28003 MR 1053796

[AS90] S. R. Adams and R. J. Spatzier, Kazhdan groups, cocycles and trees. Amer. J. Math. 112 (1990), 271-287. Zbl 0712.22007 MR 1047300

[Alv08b] A. Alvarez, Une théorie de Bass-Serre pour les relations d'équivalence et les groupoïdes boréliens. Preprint 2008.

[Alv08a] A. Alvarez, Théorème de Kurosh pour les relations d'équivalence boréliennes. Ann. Inst. Fourier (Grenoble) 60 (2010), 1161-1200. Zbl 05793928 MR 2722237

[BV97] M. E. B. Bekka and A. Valette, Group cohomology, harmonic functions and the first $L^{2}$-Betti number. Potential Anal. 6 (1997), 313-326. Zbl 0882.22013 MR 1452785

[CG86] J. Cheeger and M. Gromov, $L_{2}$-cohomology and group cohomology. Topology 25 (1986), 189-215. Zbl 0597.57020 MR 837621

[CH10] I. Chifan and C. Houdayer, Bass-Serre rigidity results in von Neumann algebras. Duke Math. J. 153 (2010), 23-54. Zbl 1201.46057 MR 2641939

[Gab98] D. Gaboriau, Mercuriale de groupes et de relations. C. R. Acad. Sci. Paris Sér. I Math. 326 (1998), 219-222. Zbl 1007.28012 MR 1646912

[Gab00] D. Gaboriau, Coût des relations d'équivalence et des groupes. Invent. Math. 139 (2000), 41-98. Zbl 0939.28012 MR 1728876

[Gab02a] D. Gaboriau, Invariants $l^{2}$ de relations d'équivalence et de groupes. Publ. Math. Inst. Hautes Études Sci. 95 (2002), 93-150. Zbl 1022.37002 MR 1953191

[Gab02b] D. Gaboriau, On orbit equivalence of measure preserving actions. In Rigidity in dynamics and geometry (Cambridge, 2000), Springer-Verlag, Berlin 2002, 167186. Zbl 1036.22008 MR 1919400

[Gab05] D. Gaboriau, Examples of groups that are measure equivalent to the free group. Ergodic Theory Dynam. Systems 25 (2005), 1809-1827.Zbl 1130.37311 MR 2183295

[Gab08] D. Gaboriau, Free product actions with relative property (T) and trivial outer automorphism groups. J. Funct. Anal. 260 (2011), 414-427. Zbl 1211.46071 MR 2737406

[Hjo06] G. Hjorth, A lemma for cost attained. Ann. Pure Appl. Logic 143 (2006), 87-102. Zbl 1101.37004 MR 2258624

[IPP08] A. Ioana, J. Peterson, and S. Popa, Amalgamated free products of weakly rigid factors and calculation of their symmetry groups. Acta Math. 200 (2008), 85-153. Zbl 1149.46047 MR 2386109

[JKL02] S. Jackson, A. S. Kechris, and A. Louveau, Countable Borel equivalence relations. J. Math. Log. 2 (2002), 1-80. Zbl 1008.03031 MR 1900547

[Kur34] A. Kurosch, Die Untergruppen der freien Produkte von beliebigen Gruppen. Math. Ann. 109 (1934), 647-660. Zbl 0009.01004 
[Lev95] G. Levitt, On the cost of generating an equivalence relation. Ergodic Theory Dynam. Systems 15 (1995), 1173-1181. Zbl 0843.28010 MR 1366313

[Lüc02] W. Lück, $L^{2}$-invariants: theory and applications to geometry and $K$-theory. Ergeb Math. Grenzgeb. (3) 44, Springer-Verlag, Berlin 2002. Zbl 1009.55001 MR 1926649

[MS06] N. Monod and Y. Shalom, Orbit equivalence rigidity and bounded cohomology. Ann. of Math. (2) 164 (2006), 825-878. Zbl 1129.37003 MR 2259246

[OW80] D. S. Ornstein and B. Weiss, Ergodic theory of amenable group actions. I: The Rohlin lemma. Bull. Amer. Math. Soc. (N.S.) 2 (1980), 161-164. Zbl 0427.28018 MR 551753

[Pop06] S. Popa, On a class of type $\mathrm{II}_{1}$ factors with Betti numbers invariants. Ann. of Math. (2) 163 (2006), 809-899. Zbl 1120.46045 MR 2215135

[Sak10] H. Sako, Measure equivalence rigidity and bi-exactness of groups. J. Funct. Anal. 257 (2009), 3167-3202. Zbl 05638309 MR 2568688

[Ser77] J.-P. Serre, Arbres, amalgames, SL 2. Astérisque 46 (1977). Zbl 0369.20013 MR 0476875

[SS88] T. A. Slaman and J. R. Steel, Definable functions on degrees. In Cabal Seminar 81-85, Lecture Notes in Math. 1333, Springer-Verlag, Berlin 1988, 37-55. Zbl 0677.03038 MR 960895

[Wei84] B. Weiss, Measurable dynamics. In Conference in modern analysis and probability (New Haven, Conn., 1982), Contemp. Math. 26, Amer. Math. Soc., Providence, RI, 1984, 395-421. Zbl 0599.28023 MR 737417

[Zim84] R. J.Zimmer, Ergodic theory and semisimple groups. Monogr. Math. 81, Birkhäuser Verlag, Basel 1984. Zbl 0571.58015 MR 776417

Received February 17, 2009; revised May 28, 2010

A. Alvarez, Université d'Orléans - UFR Sciences, Bâtiment de Mathématiques, Route de Chartres, B.P. 6759, 45067 Orléans cedex 2, France

E-mail: aurelien.alvarez@univ-orleans.fr

D. Gaboriau, Université de Lyon - CNRS, ENS-Lyon, UMPA UMR 5669, 69364 Lyon cedex 7, France

E-mail: damien.gaboriau@ens-lyon.fr 